\title{
The slow motion of slender rod-like particles in a second-order fluid
}

\author{
By L. G. LEAL \\ Chemical Engineering, California Institute of Technology, Pasadena
}

(Received 16 March 1974 and in revised form 10 June 1974)

\begin{abstract}
The motion of a slender axisymmetric rod-like particle is investigated theoretically for translation through a quiescent second-order fluid and for rotation in a simple shear flow of the same material. The analysis consists of an asymptotic expansion about the limit of rheologically slow flow, coupled with an application of a generalized form of the reciprocal theorem of Lorentz to calculate the force and torque on the particle. It is shown that an arbitrarily oriented particle with fore-aft symmetry translates, to a first approximation, at the same rate as in an equivalent Newtonian fluid, but that the motion of particles with no fore-aft symmetry may be modified at the same level of approximation. In addition, it is found that freely translating particles with fore-aft symmetry exhibit a single stable orientation with the axis of revolution vertical. In simple shear flow at small and moderate shear rates, the non-Newtonian nature of the suspending fluid causes a drift through Jeffery orbits to the equilibrium orbit $C=0$ in which the particle rotates about its axis of revolution. At larger shear rates, the particle aligns itself in the direction of flow and ceases to rotate. Comparison with the available experimental data indicates that the measured rate of orbit drift may be used to determine the second normal stress difference parameter of the secondorder fluid model. Finally, in an appendix, some preliminary observations are reported of the motion of slender rod-like particles falling thpough a quiescent viscoelastic fluid.
\end{abstract}

\section{Introduction}

It is becoming apparent that the motion of a submerged body in a viscoelastic ambient fluid is often fundamentally different from its motion in a Newtonian fluid. Examples of such differences include the lateral migration of rigid particles in nonlinear shear flow at very small Reynolds number (Karnis \& Mason 1967), the drift of rigid non-spherical particles towards preferred equilibrium orbits in simple shear flow (Gauthier, Goldsmith \& Mason 1971) and the existence, at larger shear rates, of an equilibrium orientation with a complete lack of rotation for slender rod-like particles and very flat disks (Bartram \& Mason 1974). Very few of these phenomena have been adequately investigated theoretically. Indeed, the only external flow solutions at present available are for simple translation and/or rotation of a rigid sphere under the simplifying approximation of rheologically slow flow (Leslie 1961; Caswell \& Schwarz 1962; Giesekus 1963), and the 
corresponding uniform flow past a circular cylinder, which was recently obtained by Mena \& Caswell (1974, private communication). The key result of these latter investigations is that both the drag and the torque exerted on the body occur at second order with respect to the ratio of the relaxation time scale of the material to the convective time scale of the motion, in spite of the fact that the velocity and pressure fields are already altered in a non-trivial way at first order.

The present paper represents an initial study whose purpose is the generalization of previous theoretical work to the case of straight slender axisymmetric particles. The analysis is based on the approximation of rheologically slow flow and therefore employs the Rivlin-Erickson $n$ th-order fluid model. Two separate cases are considered: translation (or sedimentation) through a quiescent ambient fluid and the rotation of a neutrally buoyant particle in simple (linear) shear flow. The chief practical interest in the sedimentation calculation is the possibility that the non-Newtonian characteristics of the ambient fluid may lead to intrinsically preferred equilibrium orientations. In addition, the analysis makes possible a partial assessment of the role of particle geometry in determining the presence or absence of first-order contributions to the hydrodynamic force and torque on the particle. The case of particle rotation in shear flow, which was first discussed (qualitatively) by Saffman (1956), is a logical first step in understanding the rheological behaviour of a dilute viscoelastic suspension of rod-like particles which is undergoing simple bulk shear flow.

The analysis is formally carried out as a perturbation expansion in the small parameter for rheologically slow flow, which is the ratio of the natural relaxation time of the ambient fluid to the convective time scale of the motion. At first order, the problem reduces to the case of Newtonian creeping flow, and in order to maintain maximum flexibility with respect to the detailed geometry, the solution is represented via the approximate slender-body theory for low Reynolds number flow (cf. Batchelor 1970; Cox 1970a). At second order, corresponding to the secondorder fluid approximation, the velocity and pressure fields may also be calculated using the slender-body approach. However, in the present work, we obtain only the force and torque on the particle, using a generalized version of the reciprocal theorem for low Reynolds number flow. As we shall demonstrate, these secondorder contributions to the force and torque can be calculated knowing only the Newtonian velocity and pressure fields.

The approximation of nearly Newtonian slow flow severely limits the magnitude of the instantaneous, non-Newtonian contributions to the particle's motion. Nevertheless, these small effects may still have a large accumulative influence on the particle orientation in sedimentation and on the orbit of rotation in shear flow. In each instance, the orientation in the Newtonian case is fully determined by the orientation of the particle at some initial time; no intrinsic preference is shown for any orientation or any orbit. In these circumstances, small nonNewtonian contributions can have a profound influence, ultimately causing the particle to attain a steady-state sedimentation orientation or a steady-state shear orbit which is completely independent of the initial state.

In $\S 2$, we define and set up the general problem in terms suitable for solution by the slow-flow perturbation expansion. This is followed in $\$ 3$ by a brief review of 
the basic Newtonian slender-body solutions for uniform translation and rotation in simple shear flow. Section 4 is concerned with the general scheme for application of the generalized reciprocal theorem to obtain the first (second-order fluid) non-Newtonian corrections for the force and torque acting on the particle. The remainder of the paper reports the application of the general formulae to calculate the hydrodynamic contributions to the force and torque acting on a rigid axisymmetric particle in the specific cases of translation with arbitrary orientation through a quiescent fluid and rotation in a simple shear flow. In the latter case, a detailed comparison is made with the recent experimental observations of Mason and co-workers (Karnis \& Mason 1967; Gauthier et al. 1971; Bartram \& Mason 1974). Finally, in an appendix we report the results of some simple observations of the translational and rotational motion of slender cylinders and circular cones falling under the action of gravity through a quiescent viscoelastic fluid.

\section{The general problem}

We consider a straight, slender, axisymmetric, rigid body of length $2 l$ which has a cross-sectional radius $R\left(x_{1}\right)$, with $x_{1}$ measured along the axis of revolution from $-l$ to $+l$. The radius $R$ is assumed to vary continuously with $x_{1}$ and to satisfy the additional constraints $R(l)=R(-l)=0$ and $R\left(x_{1}\right) / 2 l \ll 1$ for $-l<x_{1}<l$. We shall use Cartesian reference axes $\left(x_{1}, x_{2}, x_{3}\right)$ fixed in the particle and denote the undisturbed flow relative to these as $-\mathbf{U}(\mathbf{x}, t)$. The time dependence of $\mathbf{U}$ will be assumed to result in the present circumstances entirely from time-dependent changes in particle orientation rather than time variations in the flow itself. The suspending fluid is assumed to be incompressible and non-Newtonian, and is modelled as a Rivlin-Erickson second-order fluid in order to be consistent with the basic assumption of rheologically slow flow. Hence, in dimensionless terms, the bulk stress is given by

$$
\mathbf{T}=-p \mathbf{I}+\mathbf{A}_{1}+\lambda\left[\left(\mathbf{A}_{1}\right)^{2}+\epsilon_{1} \mathbf{A}_{2}\right]+O\left(\lambda^{2}\right),
$$

with $\mathbf{A}_{n}$ representing the $n$ th-order Rivlin-Erickson tensor. The components of $\mathbf{A}_{1}$ and $\mathbf{A}_{2}$, respectively, are

and

$$
a_{i j}^{(1)} \equiv u_{i, j}+u_{j, i}
$$

$$
a_{i j}^{(2)}=d a_{i j}^{(1)} / d t+u^{k} a_{i j, k}^{(1)}+a_{i k}^{(1)} u^{k, j}+a_{k j}^{(1)} u^{k},{ }_{i}
$$

The dimensionless parameters $\lambda$ and $\epsilon_{1}$ are defined as $\Phi_{3} U / \mu l$ and $\Phi_{2} / \Phi_{3}$, in which $\mu$ is the zero-shear viscosity, $\Phi_{2}$ and $\Phi_{3}$ the zero-shear normal stress coefficients and $U$ a characteristic velocity scale based on the undisturbed velocity field.

The dimensionless parameter $\lambda$ is a measure of the intrinsic relaxation time for the fluid relative to the dynamic scale $l / U$. In the present work we consider $\lambda$ to be small so that the constitutive relationship (1) differs only slightly from that for a Newtonian fluid. It is in this sense that we call the bulk motion rheologically slow. In addition, we assume that the fluid motion is also dynamically slow so that inertial effects may be neglected. More precisely, we assume that $R e \ll \lambda \ll 1$ 
so that the creeping-motion velocity and pressure fields are primarily modified by nonlinear effects associated with non-zero values of $\lambda$ rather than dynamic inertia effects associated with non-zero values of the Reynolds number. Hence we seek solutions for $\mathbf{u}$ and $p$ in the asymptotic form

$$
\left.\begin{array}{l}
\mathbf{u}=\mathbf{u}_{0}+\lambda \mathbf{u}_{1}+\ldots \\
p=p_{0}+\lambda p_{1}+\ldots
\end{array}\right\} \text { as } \lambda \rightarrow 0 .
$$

Substituting these expansions into the equations of motion and continuity, neglecting the inertia terms and equating like powers of $\lambda$ in the remaining terms, we obtain the governing equations at $O(1)$ and at $O(\lambda)$ :

$$
\begin{gathered}
\nabla^{2} \mathbf{u}_{0}-\nabla p_{0}=0, \quad \nabla \cdot \mathbf{u}_{0}=0, \\
\nabla^{2} \mathbf{u}_{1}-\nabla p_{1}=-\nabla \cdot\left[\left(\mathbf{A}_{1}\right)^{2}+\epsilon_{1} \mathbf{A}_{2}\right]_{\mathbf{u}=\mathbf{u}_{0}}, \quad \nabla \cdot \mathbf{u}_{\llcorner}=\mathbf{0} .
\end{gathered}
$$

The symbol [ $]_{\mathfrak{n}=\mathbf{u}_{0}}$ in (4) signifies that the included expression is to be evaluated using the zeroth-order velocity field $\mathbf{u}_{0}$. It is convenient to express $\mathbf{u}_{0}$ as the sum of the undisturbed velocity field $-\mathbf{U}(\mathbf{x}, t)$ and a disturbance field $+\mathbf{v}^{(0)}(\mathbf{x}, t)$. Provided that $\mathbf{U}(\mathbf{x}, t)$ satisfies (3), as we shall assume, the Newtonian contribution to the disturbance velocity field is obtained as the solution of the problem

$$
\nabla^{2} \mathbf{v}^{(0)}-\nabla \hat{p}^{(0)}=0, \quad \nabla \cdot \mathbf{v}^{(0)}=0
$$

with boundary conditions

$$
\mathbf{v}^{(0)} \rightarrow\left\{\begin{array}{l}
0 \text { as } \quad r \rightarrow \infty, \\
+\mathbf{U}(\mathbf{x}, t) \text { for } \mathbf{x} \text { on the surface } S \text { of the particle. }
\end{array}\right\}
$$

\section{The Newtonian solutions}

The linear problem posed by (5) and (6) is solved most conveniently, for the present purposes, using the standard methods of slender-body theory for low Reynolds number flows. In this approach, an approximation to the disturbance flow is obtained by modelling the effect of the actual particle by a distribution of force, source, force-dipole and higher-order singularities spread along the particle's axis of revolution. When the undisturbed velocity field evaluated along this axis is non-zero, as we shall assume, the disturbance velocity field is dominated by the force (Stokeslet) singularities. The unknown distribution of Stokeslet strength is determined by the requirement that $-\mathbf{v}^{(\mathbf{0})}+\mathbf{U}$ be zero at the body surface. The resulting (dimensionless) zeroth-order velocity field, in terms of the Cartesian co-ordinates fixed in the particle, can be expressed as

$$
\left(u_{0}\right)_{i}=-U_{i}(\mathbf{x}, t)+\frac{1}{8 \pi} \int_{-1}^{1}\left[\frac{F_{i}\left(x^{\prime}, t\right)}{|x|}+\frac{\left(x_{i}-x_{i}^{\prime}\right)\left(x_{j}-x_{j}^{\prime}\right) F_{j}\left(x^{\prime}, t\right)}{|x|^{3}}\right] d x^{\prime}
$$

in which $\mathbf{F}\left(x^{\prime}, t\right)$ is the line density of Stokeslet strength non-dimensionalized with respect to $\mu U, x_{i}^{\prime}=\delta_{i 1} x^{\prime}$ and $|x| \equiv\left\{r^{2}+\left(x_{1}-x^{\prime}\right)^{2}\right\}^{\frac{1}{2}}$, with $r=\left(x_{2}^{2}+x_{3}^{2}\right)^{\frac{1}{2}}$. As we have already noted, the dependence on $t$ occurs because of the rotation of the particle, and hence of the Cartesian co-ordinates which are fixed in the particle, with respect to fixed laboratory co-ordinates in which the undisturbed velocity field is assumed to be steady. The specific form of $F_{i}$ depends both on the form of 


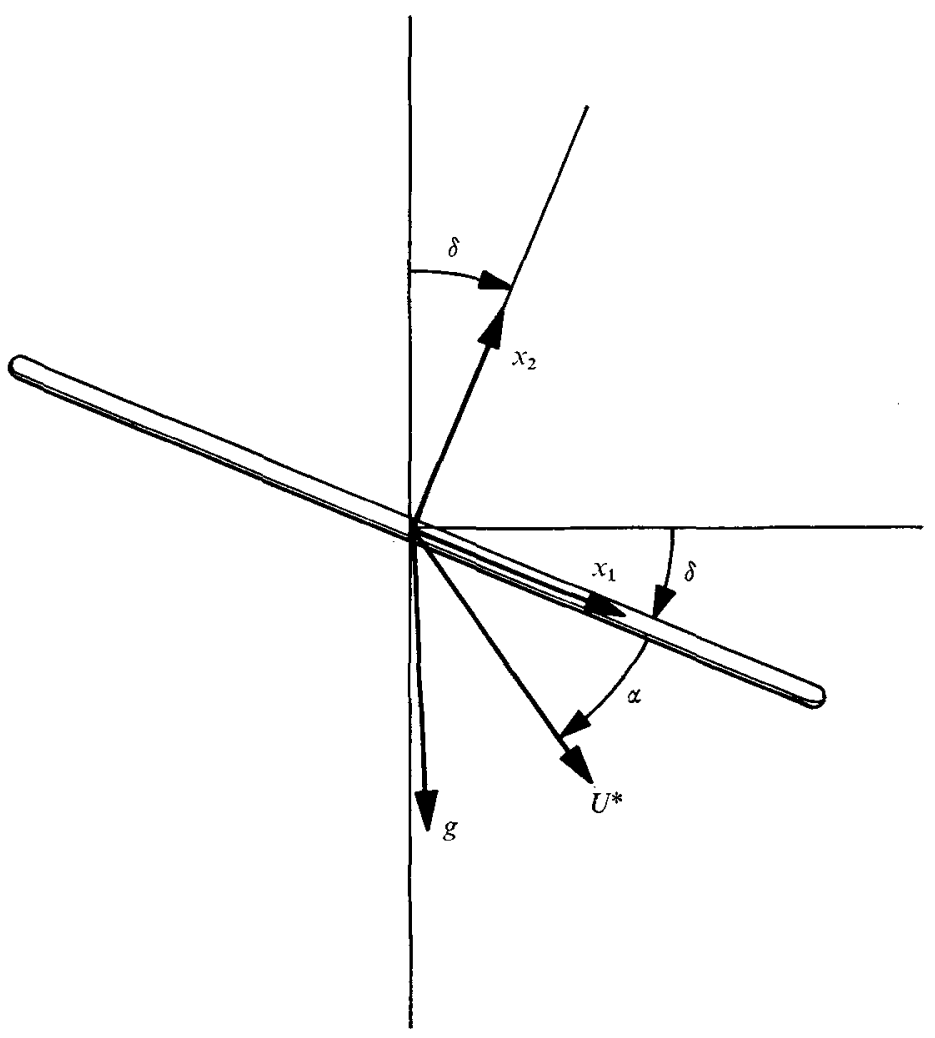

FIgURE 1. A typical axisymmetric particle sedimenting through a quiescent fluid.

the undisturbed field evaluated on the particle axis and on the detailed particle geometry. However, Batchelor (1970), Cox (1970a), Tillett (1970) and others have shown that the Stokeslet strength distribution can be expressed as an infinite series in increasing powers of $(\ln \epsilon)^{-1}$ :

$$
F_{i}=(\ln \epsilon)^{-1} f_{i}^{(0)}+(\ln \epsilon)^{-2} f_{i}^{(1)}+\ldots,
$$

where $\epsilon\left(\equiv R_{0} / 2 l\right)$ is the small parameter of slender-body analysis. Here $R_{0}$ is a length scale representative in some way of the values of $R$ over the length of the body. Higher-order singularities and further corrections to the Stokeslet distribution occur at $O\left(\epsilon^{2}\right)$. The functions $f_{i}^{(0)}, f_{i}^{(1)}$ and $f_{i}^{(2)}$ have been evaluated by Cox $(1970 a)$ for a general linear undisturbed velocity distribution. Most significant is the result, also given by Batchelor and others, that the dominant term in the Stokeslet expansion is independent of the detailed particle geometry. Results of considerable generality with respect to particle geometry are therefore possible.

When - $U$ is a vector of uniform direction and constant magnitude along the particle's axis of revolution, it is easily shown (cf. Batchelor 1970) that

$$
f_{1}^{(0)}=2 \pi U_{1}, \quad f_{i}^{(0)}=4 \pi U_{i} \quad(i=2,3) .
$$

This is the case relevant to the sedimentation of an arbitrarily oriented particle without rotation at a velocity $\mathbf{U}^{*}$ through an otherwise quiescent fluid as sketched 


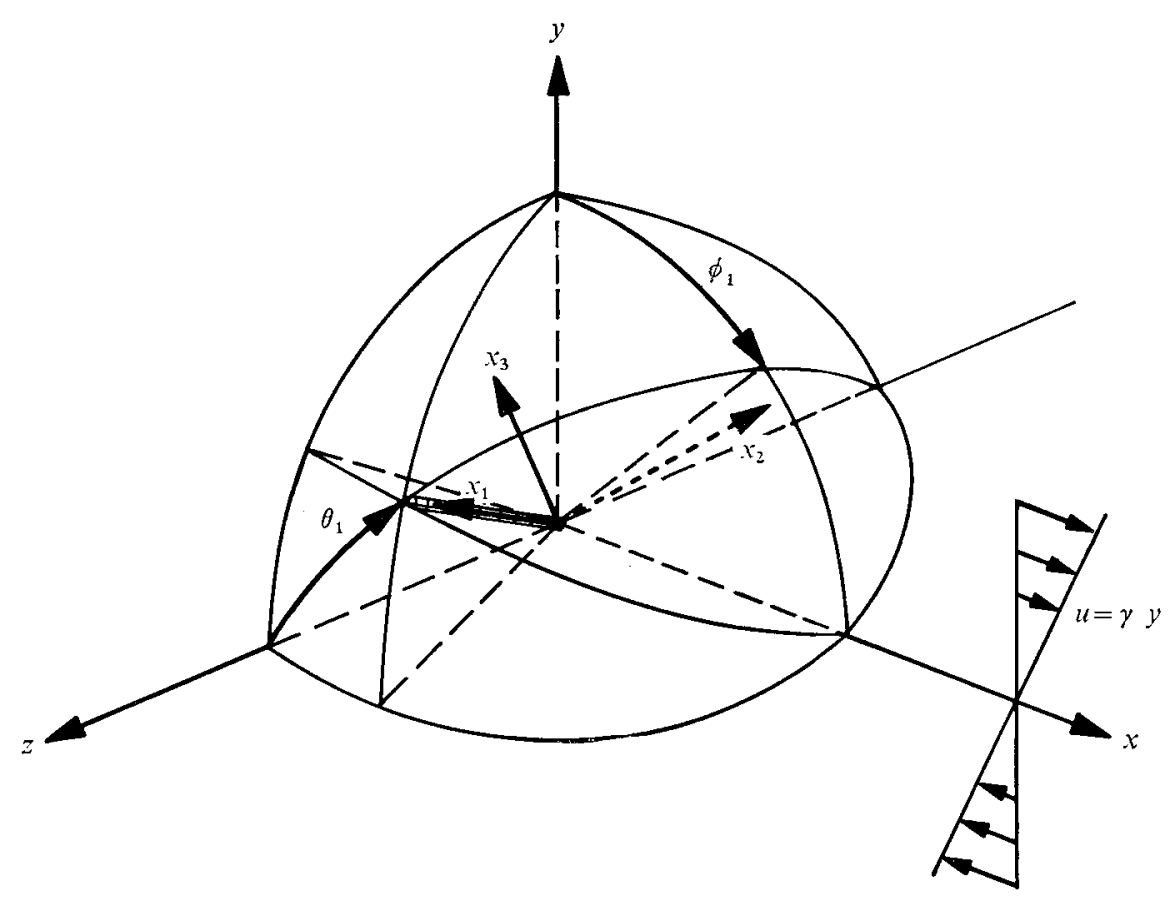

Figure 2. A typical axisymmetric, neutrally buoyant particle subjected to a simple linear shear flow.

in figure 1 . The velocity components $U_{i}$, non-dimensionalized by the characteristic velocity $U \equiv U^{*}$, are simply

$$
U_{1}=\cos \alpha, \quad U_{2}=-\sin \alpha .
$$

The second case of interest in the present context is the rotation of a neutrally buoyant axisymmetric particle in the undisturbed linear shear flow

$$
\mathbf{V}=y \mathbf{i}
$$

expressed relative to a fixed laboratory co-ordinate system $(x, y, z)$ and nondimensionalized with respect to the characteristic velocity scale $U=\gamma l$, in which $\gamma$ is the shear rate. As shown in figure 2, the particle orientation is completely specified, relative to this fixed system, by the polar angles $\theta_{1}$ and $\phi_{1}$ of the axis of revolution. For convenience, the instantaneous particle-fixed co-ordinates $\left(x_{1}, x_{2}, x_{3}\right)$, also shown in figure 2 , are chosen such that

$$
\mathbf{i}_{3}=\mathbf{k} \times \mathbf{i}_{1}, \quad \mathbf{i}_{2}=-\mathbf{i}_{1} \times \mathbf{i}_{3} .
$$

The symmetry axis of the particle is assumed to be rotating with dimensionless angular velocities $\omega_{2}$ and $\omega_{3}$ as a result of the interactions of the particle with the surrounding suspending fluid. Hence the undisturbed velocity field (11), re-expressed in the particle-fixed co-ordinates, is simply

$$
U_{i}=A_{i j} x_{j} \quad(i=1,2 \text { or } 3),
$$


where the matrix $\mathbf{A}$ is defined by

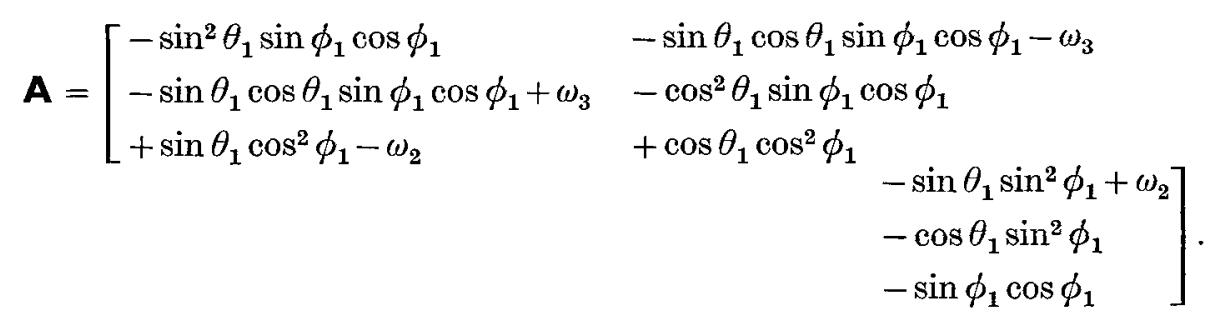

Along the particle's axis of revolution $\left(x_{2}=x_{3}=0,-1 \leqslant x_{1} \leqslant 1\right)$ the undisturbed velocity distribution is thus

$$
\left.\begin{array}{l}
U_{1}=-\sin ^{2} \theta_{1} \sin \phi_{1} \cos \phi_{1} x_{1}, \\
U_{2}=\left(-\sin \theta_{1} \cos \theta_{1} \sin \phi_{1} \cos \phi_{1}+\omega_{3}\right) x_{1}, \\
U_{3}=\left(+\sin \theta_{1} \cos ^{2} \phi_{1}-\omega_{2}\right) x_{1} .
\end{array}\right\}
$$

For the general case

$$
\left.\mathbf{U}(\mathbf{x}, t)\right|_{\mathbf{x}=\mathbf{R}}=\mathbf{U}\left(x_{1}, t\right)=\left[a(t) x_{1}, b(t) x_{1}, c(t) x_{1}\right],
$$

of which (13) is a specific example, Batchelor (1970) has shown that the dominant coefficients $f_{i}^{(0)}$ of the expansion (8) are

$$
f_{1}^{(0)}\left(x^{\prime} t\right)=2 \pi a(t) x^{\prime}, \quad f_{2}^{(0)}\left(x^{\prime}, t\right)=4 \pi b(t) x^{\prime}, \quad f_{3}^{(0)}\left(x^{\prime}, t\right)=4 \pi c(t) x^{\prime} .
$$

The coefficients $a(t), b(t)$ and $c(t)$ are dependent on time owing to the implicit time dependence of $\theta_{1}, \phi_{1}, \omega_{2}$ and $\omega_{3}$.

The analysis which follows will consider only the non-Newtonian effects resulting from the disturbance velocity field generated by this lowest-order geometryindependent approximation (15) of the Stokeslet distribution. An exceptional case in which the first contributions to the distribution of singularities along the particle's axis are $O\left(\epsilon^{2}\right)$, rather than $O(\ln \epsilon)^{-1}$, occurs when the undisturbed velocity vector vanishes identically: $a(t) \equiv b(t) \equiv c(t) \equiv 0$. It will be shown later that

$$
\omega_{3}=\sin \theta_{1} \cos \theta_{1} \sin \phi_{1} \cos \phi_{1}+O(\lambda) \text { and } \omega_{2}=\sin \theta_{1} \cos ^{2} \phi_{1}+O(\lambda),
$$

so that $b=c=0$ to first order in $\lambda$. Thus the practical significance of the exceptional case is the condition $\left|\phi_{1}-\frac{1}{2} \pi\right|>O\left(\epsilon^{2} \ln \epsilon\right)$, which must be satisfied in order that the dominant singularity is the Stokeslet distribution (15), as we have implicitly assumed. In the present work, we limit our considerations to situations in which this condition is satisfied.

\section{The first non-Newtonian contributions to the force and torque on the particle}

In this section we consider the method of calculating the first ('second-order fluid') non-Newtonian contributions to the force and torque acting on the particle. Rather than attempting to evaluate these quantities directly by solving (4) for the corresponding $O(\lambda)$ velocity and pressure fields, we use the generalized 
reciprocal theorem of Lorentz. We shall see that this method provides the $O(\lambda)$ contribution to the force and torque on each particle without the need for an explicit solution for $\mathbf{u}_{1}$ or $p_{1}$. A general description of the method has been given by Cox \& Brenner (1968) and, more recently, by Ho \& Leal (1974).

We begin by defining the tensor

$$
\tau^{(\mathbf{1})}=-p_{1} \mathbf{l}+\nabla \mathbf{u}_{1}+\left(\nabla \mathbf{u}_{1}\right)^{T}
$$

in which $p_{1}$ and $\mathbf{u}_{1}$ are the $O(\lambda)$ contributions to the pressure and velocity fields near a particle. In terms of $\tau^{(1)}$, the governing equation (4) is simply

$$
\begin{gathered}
\boldsymbol{\tau}_{j l, j}^{(1)}+H_{l}=0, \\
\text { with } \quad H_{i} \equiv \partial\left[\left(\mathbf{A}_{1}^{(0)}\right)^{2}+\epsilon_{1} \mathbf{A}_{2}^{(0)}\right]_{j l} / \partial x_{j},
\end{gathered}
$$

and the $O(\lambda)$ contribution to the hydrodynamic force on the particle is given by

$$
F_{j}^{(1)}=\mu U l \int_{S} n_{i}\left[\tau^{(\mathbf{1})}+\left(\mathbf{A}_{1}^{(0)}\right)^{2}+\epsilon_{\mathbf{1}} \mathbf{A}_{\mathbf{2}}\right]_{i j} d A
$$

Here, $\mathbf{n}$ is the outward normal to the particle and integration is over its surface. The superscript (0) on $\left(\mathbf{A}_{1}\right)^{2}$ and $\mathbf{A}_{2}$ in both (18) and (19) signifies that these quantities are to be evaluated using the $O(1)$ velocity field $\mathbf{u}_{0}$. In view of the definition (16), evaluation of $F_{j}^{(1)}$ using (19) would appear to require an explicit knowledge of the $O(\lambda)$ velocity and pressure fields. However, using the reciprocal theorem, it is straightforward to show (see Cox \& Brenner 1968; Ho \& Leal 1974) that

$$
\int_{S} n_{i} \tau_{i j}^{(1)} d A \equiv \int_{\nabla_{f}}\left(w_{j k} H_{k}\right) d Q
$$

in which $V_{f}$ is the whole volume of fluid surrounding the particle. As we have already implied, the function $H_{k}$ depends only on the $O(1)$ components of the velocity field. The tensor function $w_{j k}$ satisfies

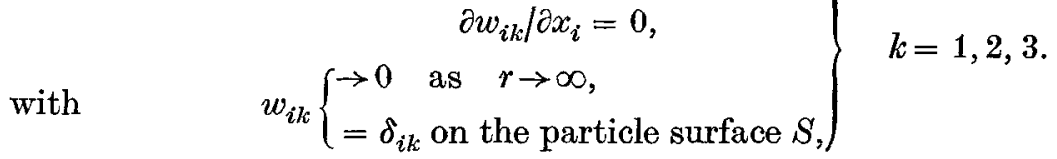

That is, for each fixed value of $k, w_{i k}$ and $q_{k}$ are the creeping-motion velocity and pressure fields resulting from simple translation of the particle with unit velocity in the $k$ direction through an otherwise quiescent Newtonian fluid. Referring to (7)-(9), the solution of (21) is simply

$$
w_{i k}=\frac{1}{8 \pi} \int_{-1}^{1}\left[\frac{\hat{F}_{i}^{k}\left(x^{\prime}, t\right)}{|x|}+\frac{\left(x_{i}-x_{i}^{\prime}\right)\left(x_{j}-x_{j}^{\prime}\right) \hat{F}_{j}^{k}\left(x^{\prime}, t\right)}{|x|^{3}}\right] d x^{\prime}
$$

with

$$
\hat{F}_{i}^{k}=\left\{\begin{array}{ll}
2 \pi(\ln \epsilon)^{-1} \delta_{i k}, & k=1, \\
4 \pi(\ln \epsilon)^{-1} \delta_{i k}, & k=2,3
\end{array}\right\}
$$


Hence, given the $O(1)$ velocity distribution $\mathbf{u}_{0}$, the function $H_{l}$ may be calculated using (18), and the force then evaluated from (19) with the first term replaced by (20) and $w_{i k}$ given by (22) and (23).

In a similar manner the torque $\mathbf{G}^{(1)}$ on the particle at $O(\lambda)$ may also be calculated from the $O(1)$ velocity and pressure fields. The basic equation for the torque is

$$
G_{i}^{(1)}=\mu U l^{2} \int_{S} \epsilon_{i j k} r_{j} n_{l}\left[\tau^{(\mathbf{1})}+\left(\mathbf{A}_{1}^{(0)}\right)^{2}+\epsilon_{1} \mathbf{A}_{2}\right]_{l k} d A,
$$

in which $\mathbf{r}$ is the position vector from the centre of the particle to its surface. In this case, the reciprocal theorem may be used to re-express the term involving $\tau_{l k}^{(1)}$ in the form

$$
\int_{S} \epsilon_{i j k} r_{j} n_{l} \tau_{i k}^{(1)} d A=-\int_{V_{f}}\left(t_{i k} H_{k}\right) d Q
$$

The function $t_{i k}$ satisfies

with

$$
\left.\begin{array}{c}
d^{2} t_{i k} / d x_{j}^{2}-d Q_{k} / d x_{j}=0, \\
d t_{i k} / d x_{i}=0, \\
\rightarrow 0 \quad \text { as } \quad r \rightarrow \infty, \\
=\epsilon_{i k j} x_{j} \text { on the body surface } S,
\end{array}\right) k=1,2,3 .
$$

For a slender body, the surface $S$ corresponds, to a first approximation, to $x_{2}=x_{3}=0$. Therefore, in this case $t_{i k} \equiv w_{i k}$, as given in (22), with

$$
\widetilde{F}_{1}^{k}=\epsilon_{i k 1}\left(4 \pi(\ln \epsilon)^{-1} \cdot x^{\prime}\right) \text {. }
$$

As before, the reciprocal theorem (25) provides a method for calculating the torque on the particle at $O(\lambda)$ from a knowledge of the pressure and velocity fields at $O(1)$.

In calculating $H_{i}$ from (18) for an arbitrary undisturbed velocity field, it is convenient to use the decomposition of $u_{0 i}$ given in (7). The various integrals with respect to $x^{\prime}$ over the range -1 to 1 which are required for the disturbance field $v_{i}^{(0)}$ and its derivatives in (18) can be evaluated analytically both for $F_{i}=$ constant and for $F_{i}=$ constant $\times x^{\prime}$. As indicated by (8), (9) and (15), these two cases are sufficient for simple translation with no rotation and for simple shear flow. As we shall see, in the present analysis we need only the limiting forms of these integrals for $r \ll 1$. These asymptotic forms are listed in tables 1 and 2 .

Finally, it is perhaps worth noting that all of the general formulae (19)-(21) and (24)-(26) are equally applicable to particles of arbitrary shape. The chief simplifications which occur for slender rod-like particles are inherent in the solution forms (7) and (22) for $u_{0 i}, w_{i k}$ and $t_{i k}$, and in the fact that the volume integrals in (20) and (25) are dominated by contributions from the region nearest the axis of the particle, specifically for $-1<x_{1}<1$ and $r \ll 1$.

\section{Translation through a quiescent fluid}

In this section we first consider the application of (19) and (20) to calculate the force acting on an arbitrarily oriented straight slender particle which is translating without rotation through a quiescent fluid under the action of an externally 


\begin{tabular}{lll} 
& & \multicolumn{1}{c}{$\begin{array}{c}\text { Asymptotic form } \\
(-1 \leqslant x \leqslant 1 ; r \ll 1\end{array}$} \\
& \multicolumn{1}{c}{ Integrand } & \multicolumn{1}{c}{ except for $r<x-1, x+1)$} \\
$A$ & $1 /|x|^{3}$ & $-\ln \left[r^{2} / 4\left(1-x^{2}\right)\right]$ \\
$B$ & $1 /|x|^{3}$ & $2 / r^{2}$ \\
$C$ & $\left(x-x^{\prime}\right) /|x|^{3}$ & $-2 x /\left(1-x^{2}\right)$ \\
$D$ & $\left(x-x^{\prime}\right)^{2} /|x|^{3}$ & $-2-\ln \left[r^{2} / 4\left(1-x^{2}\right)\right]$ \\
$E$ & $1 /|x|^{5}$ & $4 / 3 r^{4}$ \\
$F$ & $\left(x-x^{\prime}\right) /|x|^{5}$ & $-\left(61 x+2 x^{3}\right) / 3\left(1-x^{2}\right)^{3}$ \\
$G$ & $\left(x-x^{\prime}\right)^{2} /|x|^{5}$ & $2 / 3 r^{2}$ \\
$H$ & $\left(x-x^{\prime}\right)^{3} /|x|^{5}$ & $-2 x /\left(1-x^{2}\right)$ \\
$I$ & $1 /|x|^{7}$ & $16 / 15 r^{6}$ \\
$J$ & $\left(x-x^{\prime}\right) /|x|^{7}$ & $-\left(101 x+201 x^{3}+2 x^{5}\right) / 5\left(1-x^{2}\right)^{5}$ \\
$K$ & $\left(x-x^{\prime}\right)^{2} /|x|^{7}$ & $4 / 15 r^{4}$ \\
$L$ & $\left(x-x^{\prime}\right)^{3} /|x|^{7}$ & $-\left(61 x+2 x^{3}\right) / 3\left(1-x^{2}\right)^{3}$ \\
$M$ & $\left(x-x^{\prime}\right)^{4} /|x|^{7}$ & $2 / 5 r^{2}$
\end{tabular}

TABLE 1. Here $|x| \equiv\left\{r^{2}+\left(x^{\prime}-x\right)^{2}\right\}^{\frac{1}{2}}, x \equiv x_{1}, x^{\prime} \equiv x_{1}^{\prime}, r^{2} \equiv x_{2}^{2}+x_{3}^{2}$,

$$
A, B, C, \ldots=\int_{-1}^{1}[\text { integrand }] d x^{\prime}
$$

applied body force. Subsequently, we investigate the stability of the various possible orientations by using (24) and (25) to determine the external torque which would be necessary to maintain the particle in a particular orientation.

\section{The force on the particle}

Batchelor (1970) has shown that the force acting on a slender particle of arbitrary cross-section translating in creeping flowthrough a Newtonian suspending fluid, as shown in figure 1 , is

$$
\left.\begin{array}{l}
F_{1}^{(0)} \sim \frac{4 \pi \mu l U^{*} \cos \alpha}{\ln \epsilon}+O\left((\ln \epsilon)^{-2}\right), \\
F_{2}^{(0)} \sim-\frac{8 \pi \mu l U^{*} \sin \alpha}{\ln \epsilon}+O\left((\ln \epsilon)^{-2}\right) .
\end{array}\right\}
$$

The first non-Newtonian contributions $F_{i}^{(1)}$ to the force are obtained from (19) and (20) in the combined form

$$
\frac{F_{j}^{(1)}}{\mu U^{* l}}=\int_{V_{f}}\left(w_{j k} H_{k}\right) d Q+\int_{S} n_{i}\left[\left(\mathbf{A}_{\mathbf{1}}^{(0)}\right)^{2}+\epsilon_{\mathbf{1}} \mathbf{A}_{2}\right]_{i j} d A
$$

The vector function $\mathbf{H}$ which appears in the first term is most conveniently expressed in the form

$$
\begin{aligned}
H_{i}=-\frac{U_{1} \epsilon_{1}}{8 \pi \ln \epsilon}\left(C_{i j} f_{j}^{(0)}\right) & -\frac{U_{2} \epsilon_{1}}{8 \pi \ln \epsilon}\left(D_{i j} f_{j}^{(0)}\right) \\
+ & \frac{1}{64 \pi^{2}(\ln \epsilon)^{2}}\left[E_{i j k} f_{j}^{(0)} f_{k}^{(0)}+\epsilon_{1} F_{i j k} f_{j}^{(0)} f_{k}^{(0)}\right]
\end{aligned}
$$


in which the coefficients $C_{i j}, D_{i j}, E_{i j k}$ and $F_{i j k}$ depend solely upon the integrals $A, B, C, \ldots, M$ (see table 1 ). $\uparrow$ Similarly, the tensoral velocity field $\mathbf{w}$ [see (22)], which is also required for the first term of (29), may be written as

$$
w_{i k}=\left(\hat{F}_{i}^{i} / 8 \pi\right) G_{i k},
$$

where $\mathbf{G}$ is the symmetric second-order tensor

$$
\mathbf{G} \equiv\left[\begin{array}{ccc}
A+D & x_{2} C & x_{3} C \\
x_{2} C & A+x_{2}^{2} B & x_{2} x_{3} B \\
x_{3} C & x_{2} x_{3} B & A+x_{3}^{2} B
\end{array}\right] .
$$

Finally, it is necessary to have definite expressions for the various components of the unit normal $\mathbf{n}$. Here, we adopt the general form

$$
n_{1}=\hat{n}_{1}\left(x_{1}\right), \quad n_{2}=\hat{n}_{2}\left(x_{1}\right) \cos \phi, \quad n_{3}=\hat{n}_{3}\left(x_{1}\right) \sin \phi,
$$

in which $\phi$ is the polar angle measured counterclockwise (towards $x_{3}$ ) from the $x_{2}$ axis (cf. figure 1). The precise form for the functions $\hat{n}_{i}\left(x_{1}\right)$ depends, of course, on the detailed geometry of the particle. However, it is possible to characterize the general properties of the $\hat{n}_{i}\left(x_{1}\right)$. In particular, provided that the particle is slender and axisymmetric as we have assumed, it may be shown that

$$
\hat{n}_{\mathbf{2}}=\hat{n}_{\mathbf{3}}=O(1)
$$

whereas $\hat{n}_{1}$ is small, $O(\epsilon)$. In addition, for particles with fore-aft symmetry, $\hat{n}_{1}$ is odd in $x_{1}$, whereas $\hat{n}_{2}$ and $\hat{n}_{3}$ are both even functions of $x_{1}$.

Combining (29), (31) and (32), the $O(\lambda)$ force contribution can finally be written in the form, suitable for computation,

$$
\frac{F_{j}^{(1)}}{\mu U^{* l}}=\int_{V_{f}} \frac{\hat{F}_{k}^{j}}{8 \pi} Y_{k} d V+\int_{S} t_{j} d A
$$

in which

$$
\begin{aligned}
& Y_{1}=(A+D) H_{1}+x_{2} C H_{2}+x_{3} C H_{3}, \\
& Y_{2}=x_{2} C H_{1}+\left(A+x_{2}^{2} B\right) H_{2}+x_{2} x_{3} B H_{3}, \\
& Y_{3}=x_{3} C H_{1}+x_{2} x_{3} B H_{2}+\left(A+x_{3}^{2} B\right) H_{3}
\end{aligned}
$$

and

$$
\begin{aligned}
t_{j}= & (\ln \epsilon)^{-2}\left\{\hat{n}_{1}\left(P_{1 j 1} f_{1}^{(0)^{2}}+P_{1 j 2} f_{1}^{(0)} f_{2}^{(0)}+P_{1 j 3} f_{2}^{(0)^{2}}\right)\right. \\
& +\hat{n}_{2} \cos \phi\left(P_{2 j 1} f_{1}^{(0)^{2}}+P_{2 j 2} f_{1}^{(0)} f_{2}^{(0)}+P_{2 j 3} f_{2}^{(0)^{2}}\right) \\
& +\hat{n}_{3} \sin \phi\left(P_{3 j 1} f_{1}^{(0)^{2}}+P_{3 j 2} f_{1}^{(0)} f_{2}^{(0)}+P_{3 j 3} f_{2}^{(0)^{2}}\right) \\
& +\epsilon_{1}\left[\hat{n}_{1}\left(Q_{1 j 1} f_{1}^{(0)^{2}}+Q_{1 j 2} f_{1}^{(0)} f_{2}^{(0)}+Q_{1 j 3} f_{2}^{(0)^{2}}\right)\right. \\
& +\hat{n}_{2} \cos \phi\left(Q_{2 j 1} f_{1}^{(0)^{2}}+Q_{2 j 2} f_{1}^{(0)} f_{2}^{(0)}+Q_{2 j 3} f_{2}^{(0)^{2}}\right) \\
& \left.\left.+\hat{n}_{3} \sin \phi\left(Q_{3 j 1} f_{1}^{(0)^{2}}+Q_{3 j 2} f_{1}^{(0)} f_{2}^{(0)}+Q_{3 j 3} f_{2}^{(0)^{2}}\right)\right]\right\}
\end{aligned}
$$

The coefficients $P_{i j k}$ and $Q_{i j k}$ are complicated functions of the various integrals of table 1 , as well as $x_{2}$ and $x_{3}$.

$\dagger$ Tables containing the specific relationships between the coefficients $C_{i j}, D_{i j}, E_{i j k}$ and $F_{i j k}$ [and $P_{i j k}$ and $Q_{i j k}$ in (35) below] and these integrals may be obtained on request from either the author or the JFM Editorial Office, Department of Applied Mathematics and Theoretical Physics, Silver Street, Cambridge CB3 9EW. 
In general, to evaluate (33) it is necessary to specify the detailed particle geometry. However, the assumed axisymmetry of the particle allows some immediate progress without specifying either the geometry or the precise functional forms of the various coefficients in $H_{i}$ and $t_{i}$. In particular, terms in the integrands in (33) which are odd in $x_{2}$ or $x_{3}$ will clearly integrate to zero for axisymmetric particles. The coefficients $A, B$ and $C$ in (34c) are independent of $x_{2}$ and $x_{3}$. On the other hand, $C_{3 i}, D_{3 i}, E_{3 i j}, F_{3 i j}, P_{1 j k}, P_{2 j k}, Q_{1 j k}$ and $Q_{2 j k}$ are all odd in $x_{3}$, while $P_{33 k}$ and $Q_{33 k}$ are even in $x_{3}$ but multiplied by $\sin \phi$ in the general relation (35). It thus follows that

$$
F_{3}^{(1)} \equiv 0
$$

for any axisymmetric particle. Hence, as expected from considerations of symmetry, an axisymmetric slender particle which is sedimenting without rotation through an otherwise quiescent fluid will experience no lateral (sideways) force regardless of its detailed geometry, or its inclination to the horizontal.

The components $F_{2}^{(1)}$ and $F_{1}^{(1)}$ are, in general, non-zero. However, for the special case of axisymmetric slender particles with fore-and-aft (end-to-end) symmetry, careful term-by-term examination of the integrands in (33) for $j=1$ and $j=2$ yields the further important result

$$
F_{1}^{(1)}=F_{2}^{(1)}=0 .
$$

For the sake of brevity, the detailed proof of (37) will be omitted. In general terms, however, it is found that those parts of the integrand in (33) which are not odd in either $x_{2}$ or $x_{3}$ are inevitably odd in $x_{1}$ and so vanish upon integration owing to the additional assumption of fore-aft symmetry. Taking (36) and (37) together, it is clear that in these circumstances there is no $O(\lambda)$ contribution to the hydrodynamic drag, in spite of the non-trivial modifications to the fluid velocity and pressure fields which occur at the same order. In this, the motion of a long slender axisymmetric particle with fore-aft symmetry is qualitatively similar to the motion of a rigid spherical particle, which was discussed in the introduction. However, this feature of the rheologically slow motion of isolated rigid particles does not carry over to the more general case of axisymmetric particles which have no fore-aft symmetry, as we shall now discuss.

When the particle does not exhibit fore-aft symmetry, it is necessary to evaluate the integrals in (33) explicitly. Although this would be difficult for particles of general shape, the task is simplified considerably for the special case of very long slender particles. The surface integrals are simplified by the fact that the integrand is evaluated at the particle surface $r_{s}=R\left(x_{1}\right) \ll 1$, where the asymptotic forms valid for $r \ll\left|\left(1 \pm x_{1}\right)\right|$ may be used for the various coefficients $P_{i j k}$ and $Q_{i j k}$. Although these asymptotic estimates generally do not hold within $O(\epsilon)$ of the particle ends, the integration over $x_{1}$ can be limited to the subdomain $-1+\epsilon \leqslant x_{1} \leqslant 1-\epsilon$ with a relative error $O(1 / \ln \epsilon)$, which is negligible at the level of approximation considered here. The volume integrals are also simplified since they are increasingly dominated by the contribution from the region

$$
-1+\epsilon<x_{1}<1-\epsilon, \quad r \ll 1
$$


as the particle becomes increasingly slender. The proof of this fact is not difficult in principle, involving only the asymptotic evaluation of the integrand for the various regions of $V_{f}$. It is sufficient for our purpose to note that the next largest contribution comes from the small region within $O(\epsilon)$ of the ends of the particle. This contribution is again asymptotically smaller than the major contribution by $O(1 / \ln \epsilon)$ for $\epsilon \ll 1$. We shall be content here to obtain only the limiting asymptotic estimate of the integrals in (33) for small $\epsilon$, which may be calculated by neglecting all contributions other than those from the subregion $-1+\epsilon \leqslant x_{1} \leqslant 1-\epsilon$, $R\left(x_{1}\right) \leqslant r<c_{1}$. The constant $c_{1}$ is kept fixed and small $(\ll 1)$, as $\epsilon \rightarrow 0$, but is otherwise arbitrary.

Hence, evaluating the integrands in (33) for $r_{s}=R\left(x_{1}\right) \ll 1$ and $r \ll\left(1-x_{1}\right)$, $\left(1+x_{1}\right)$, respectively, and integrating over $\phi$ from 0 to $2 \pi$, we obtain the following asymptotic results:

$$
\begin{aligned}
\frac{64 \pi F_{1}^{\prime 1)}}{\mu U^{* l}} \sim & \frac{1}{8 \pi(\ln \epsilon)^{2}}\left[-384 \hat{F}_{1}^{1} f_{1}^{(0)^{2}}-32 \epsilon_{1} \hat{F}_{1}^{1} f_{2}^{(0)^{2}}\right] \int_{-1+\varepsilon}^{1-\epsilon} d x_{1} \int_{R\left(x_{1}\right)}^{c_{1}} r d r \\
& \times\left[\frac{x_{1}}{r^{2}\left(1-x_{1}^{2}\right)} \ln \left(\frac{r^{2}}{4\left(1-x_{1}^{2}\right)}\right)\right]-\frac{8 \hat{F}_{1}^{1} f_{2}^{(0)} U_{2} \epsilon_{1}}{\ln \epsilon} \int_{-1+\epsilon}^{1-\epsilon} d x_{1} \int_{R\left(x_{1}\right)}^{c_{1}} r d r\left[\frac{x_{1}}{r^{2}\left(1-x_{1}^{2}\right)}\right] \\
& +\int_{-1+\epsilon}^{1-\epsilon}\left[\left(\frac{32 \hat{n}_{1}}{R^{2}\left(x_{1}\right)}-\frac{32 x_{1} \hat{n}_{2}}{R\left(x_{1}\right)\left(1-x_{1}^{2}\right)}+\frac{4 \epsilon_{1} \hat{n}_{1}}{R^{2}\left(x_{1}\right)} \ln \frac{R^{2}\left(x_{1}\right)}{4\left(1-x_{1}^{2}\right)}-\frac{96 x_{1} \epsilon_{1} \hat{n}_{2}}{R\left(x_{1}\right)\left(1-x_{1}^{2}\right)}\right)\right. \\
& \times \frac{f_{1}^{(0)}}{(\ln \epsilon)^{2}}-\left(\frac{8 R\left(x_{1}\right)\left(6 x_{1}+2 x_{1}^{3}\right) \hat{n}_{2}}{\left(1-x_{1}^{2}\right)^{3}}\right) \frac{f_{2}^{(0)}}{(\ln \epsilon)^{2}} \\
+ & \left.\frac{8 \pi \epsilon_{1}}{\ln \epsilon}\left[\frac{16 x_{1}}{1-x_{1}^{2}} \frac{d f_{1}^{(0)}}{d t} \hat{n}_{1}-\frac{8}{R\left(x_{1}\right)} \frac{d f_{2}^{(0)}}{d t} \hat{n}_{2}\right]\right] R\left(x_{1}\right) d x_{1}, \\
\frac{64 \pi F_{2}^{(1)} \sim}{\mu U^{*} l} \sim & \left.\frac{1}{8 \pi(\ln \epsilon)^{2}}\left[\left(336 \epsilon_{1}+8\right) \hat{F}_{2}^{2} f_{1}^{(0)} f_{2}^{(0)}\right]\right]_{-1+\epsilon}^{1-\epsilon} d x_{1} \\
& \times \int_{R\left(x_{1}\right)}^{c_{1}} r d r\left[\frac{x_{1}}{r^{2}\left(1-x_{1}^{2}\right)} \ln \left(\frac{r^{2}}{4\left(1-x_{1}^{2}\right)}\right)\right] \\
& +\int_{-1+\epsilon}^{1-\epsilon}\left\{\left[\frac{16 \hat{n}_{1}}{R^{2}\left(x_{1}\right)}+\frac{64 x_{1} \hat{n}_{2}}{R\left(x_{1}\right)\left(1-x_{1}^{2}\right)}-\frac{4 \epsilon_{1} \hat{n}_{1}}{R^{2}\left(x_{1}\right)}\left(\ln \frac{R^{2}\left(x_{1}\right)}{4\left(1-x_{1}^{2}\right)}+6\right)\right.\right. \\
& \left.\left.\quad \frac{44 x_{1} \hat{n}_{2}}{R\left(x_{1}\right)\left(1-x_{1}^{2}\right)}\right] \frac{f_{1}^{(0)} f_{2}^{(0)}}{(\ln \epsilon)^{2}}-\frac{32 \pi \epsilon_{1} \hat{n}_{2}}{R\left(x_{1}\right) \ln \epsilon} \frac{d f_{2}^{(0)}}{d t}\right\} R\left(x_{1}\right) d x_{1} .
\end{aligned}
$$

In order to simplify the integrands, we have used the relationships $\hat{n}_{2}=\hat{n}_{3}$, $x_{2}=r \cos \phi$ and $x_{3}=r \sin \phi$. To proceed further, we must specify $R\left(x_{1}\right)$.

Here, we briefly consider only the single example of the cone-shaped particle sketched in figure 3 , for which

$$
R\left(x_{1}\right) \sim \epsilon\left(x_{1}+1\right)
$$

and $\epsilon \equiv \frac{1}{2} R_{0}$, in the range $-1+\epsilon \leqslant x_{1} \leqslant 1-\epsilon$. Using this shape function together with the equations (9) and (10) for $f_{1}^{(0)}$ and $f_{2}^{(0)}$, and the expressions (23) for $\hat{F}_{1}^{1}$ and $\hat{F}_{2}^{2}$, equations (38) and (39) yield the asymptotic results

$$
\begin{aligned}
& F_{1}^{(1)}=\pi \mu U^{*} l\left[-2 \cos ^{2} \alpha-\epsilon_{1}\left(\frac{5}{3} \sin ^{2} \alpha\right)\right]+O(1 / \ln \epsilon), \\
& F_{2}^{(1)}=\pi \mu U^{*} l\left[-7 \epsilon_{1}-\frac{1}{6}\right] \sin \alpha \cos \alpha+O(1 / \ln \epsilon) .
\end{aligned}
$$




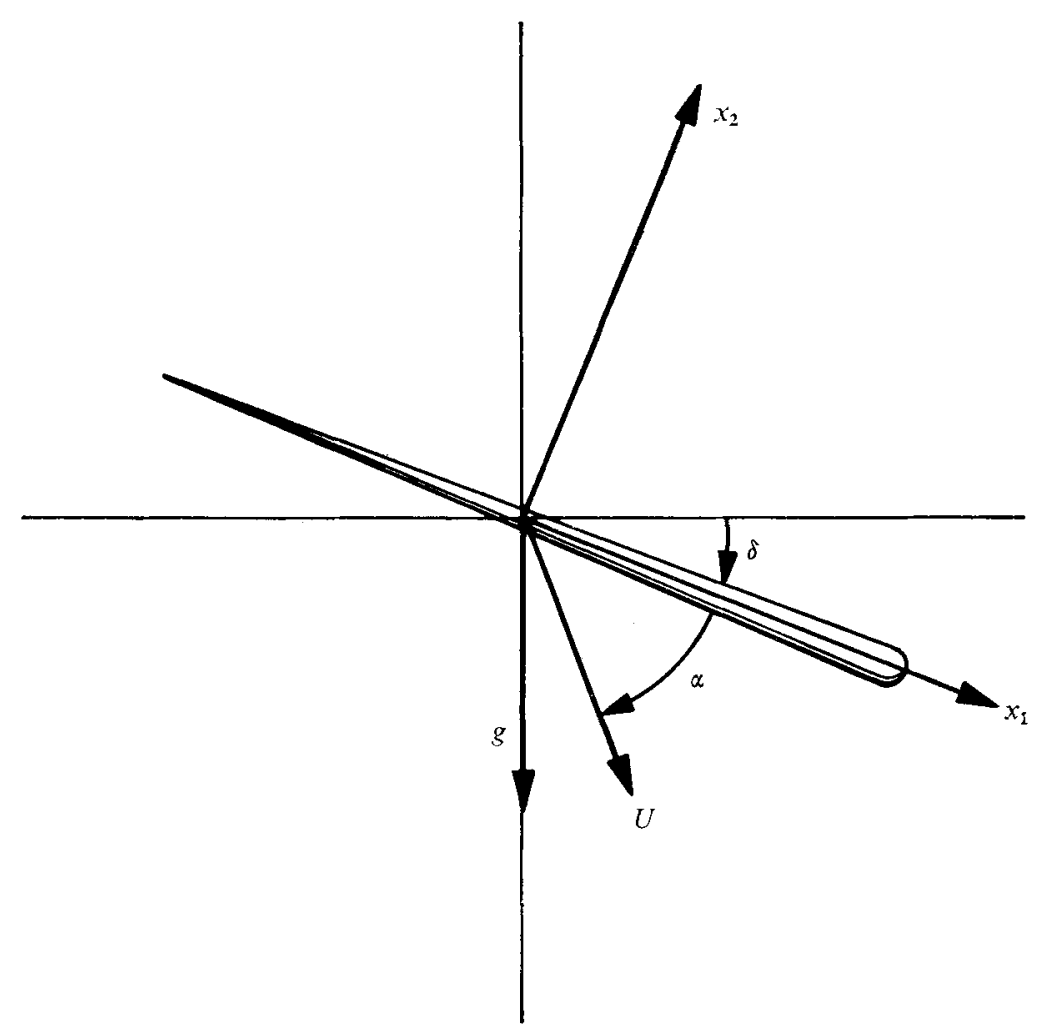

FIGURE 3. A circular conical particle sedimenting through a quiescent fluid.

Thus, if the cone axis is vertical, so that $\alpha=0$, the contributions $F_{2}^{(0)}$ and $F_{2}^{(1)}$ vanish identically, while

$$
F_{1}=4 \pi \mu l U^{*} / \ln \epsilon-2 \pi \mu U^{*} l \lambda+O\left(\lambda^{2}\right)
$$

For most viscoelastic fluids, measurements indicate that $\lambda>0$ and $\lambda \epsilon_{1}<0$ with $\left|\epsilon_{1}\right| \sim 0.5$ (cf. Caswell \& Schwarz 1962). Hence, the drag force is increased by the non-Newtonian contribution when the particle translates with the sharp end trailing. With the sharp end leading, $\alpha=\pi$ and

$$
F_{1}=-\left[4 \pi \mu l U^{*} / \ln \epsilon+2 \pi \mu U^{*} l \lambda\right]+O\left(\lambda^{2}\right) .
$$

Hence, in this case the drag force is decreased. In contrast, when the cone axis is horizontal, so that $\alpha=\frac{1}{2} \pi$, the non-Newtonian contribution $F_{2}^{(1)}$ in the direction of motion vanishes identically. On the other hand, $F_{1}^{(1)}$, corresponding to a sideways 'drift' in the direction parallel to the axis of revolution, has a non-zero first-order non-Newtonian contribution:

$$
F_{1}=\lambda F_{1}^{(1)}+O\left(\lambda^{2}\right) \sim-\frac{5}{3} \epsilon_{1} \lambda \pi \mu U^{*} l+O\left(\lambda^{2}\right) .
$$

If $\lambda \epsilon_{1}<0$, as indicated by experimental measurements, then $F_{1}>0$ for motion either upwards $\left(\alpha=-\frac{1}{2} \pi\right)$ or downwards $\left(\alpha=\frac{1}{2} \pi\right)$. Thus, a freely suspended horizontal particle would experience a slow lateral drift in the direction of the blunt end. 


\section{The torque on the particle}

The hydrodynamic force on a translating, but non-rotating axisymmetric particle depends on its orientation at both $O(1)$ and $O(\lambda)$. Thus it is important to determine whether the particle has a preferred steady-state orientation. One means of obtaining this information is to calculate the torque $-\mathbf{G}$ at each possible orientation which must be applied to keep the particle from rotating. In a Newtonian fluid at zero Reynolds number, the result is $\mathbf{G} \equiv 0$. Thus, in a Newtonian fluid the particle experiences no hydrodynamically induced torque and its orientation is fixed for all time by its initial orientation: no orientation is intrinsically preferred over any other.

The first non-Newtonian contributions to the torque are obtained from (24) and (25):

$$
\frac{G_{i}^{(1)}}{\mu U^{*} l^{2}}=\int_{V_{f}}-\left(t_{i k} H_{k}\right) d V+\int_{S} \epsilon_{i j k} r_{j} n_{l}\left[\left(\mathbf{A}_{1}^{(0)}\right)^{2}+\epsilon \mathbf{A}_{2}^{(0)}\right]_{l k} d A
$$

The general form for $H_{i}$ is again given by (30), while $t_{i k}$ is obtained from (22) $\left(t_{i k} \equiv w_{i k}\right)$ with the Stokeslet distribution $\tilde{F}_{i}^{k}$ [equation (27)] substituted for $\hat{F}_{i}^{k}$. The position vector $\mathbf{r}$ expressed in terms of the particle-fixed co-ordinates is

$$
\mathbf{r}=x_{1} \mathbf{i}_{1}+R\left(x_{1}\right) \cos \phi \mathbf{i}_{2}+R\left(x_{1}\right) \sin \phi \mathbf{i}_{3} .
$$

As in the case of the force, the general symmetry properties of the integrands in (42) allow some progress toward calculation of $G_{i}^{(1)}$ for axisymmetric particles without explicit specification of the particle geometry.

In particular, systematic evaluation of the integrands in (42) for both $i=1$ and $i=2$ shows that every term is odd in either $x_{2}$ or $x_{3}$ (i.e. $\cos \phi$ or $\sin \phi$ ). Thus, for all axisymmetric particles,

$$
G_{1}^{(1)}=G_{2}^{(1)}=0
$$

Thus, as expected from considerations of symmetry, a general axisymmetric particle translating through a quiescent second-order fluid shows no tendency, at the second-order fluid level, either to rotate about its axis of revolution or to rotate out of its initial plane of motion (i.e. the $x_{1}$, $\mathrm{U}$ plane).

The component $G_{\mathbf{3}}^{(1)}$ is given by

$$
\begin{aligned}
\frac{G_{3}^{(1)}}{\mu \bar{U}^{*} l^{2}}=\int_{V_{f}}-\left[\left(\frac{\tilde{F}_{3}^{2}}{x^{\prime}}\right) \frac{\bar{A}+x_{3}^{2} \bar{B}}{8 \pi} H_{2}\right. & \left.+\left(\frac{\widetilde{F}_{2}^{3}}{x^{\prime}}\right) \frac{x_{2} x_{3} \bar{B}}{8 \pi} H_{3}\right] d V \\
& +\int_{S}\left[-R \cos \phi t_{1}+x_{1} t_{2}\right] d A
\end{aligned}
$$

The general form of the vector function $t$ is unchanged from that in (35), however the integrals $A, \ldots, M$ (table 1 ) which originally appeared in the coefficients $P_{i j k}$ and $Q_{i j k}$ must be replaced in this case by the integrals $\bar{A}, \ldots, \bar{M}$ (table 2 ). The coefficients $\bar{A}$ and $\bar{B}$ are evaluated in table 2 . In this case, not all of the terms are odd in $x_{2}$ or $x_{3}$, and $G_{3}^{(1)}$ is non-zero for a general axisymmetric particle. Nevertheless, some simplification of (45) is still possible for axisymmetric particles. In particular, all the terms involving $f_{1}^{(0)^{2}}, f_{2}^{(0)^{2}}, C_{22}, D_{22}, C_{31}$ and $D_{32}$ are odd in 


\begin{tabular}{lll} 
& & \multicolumn{1}{c}{ Asymptotic form } \\
& \multicolumn{1}{c}{ Integrand } & \multicolumn{1}{c}{$\quad$ for $r<x-1, x+1)$} \\
& \multicolumn{1}{c}{ for $x \leqslant 1, r \ll 1$ except } \\
$\bar{A}$ & $x^{\prime} /|x|^{2}$ & $-x \ln \left[r^{2} / 4\left(1-x^{2}\right)\right]$ \\
$\bar{B}$ & $\left.x^{\prime}|| x\right|^{3}$ & $2 x / r^{2}$ \\
$\bar{C}$ & $x^{\prime}\left(x-x^{\prime}\right) /|x|^{3}$ & $-2-\ln \left[r^{2} / 4\left(1-x^{2}\right)\right]$ \\
$\bar{D}$ & $x^{\prime}\left(x-x^{\prime}\right)^{2} /|x|^{3}$ & $-x \ln \left[r^{2} / 4\left(1-x^{2}\right)\right]$ \\
$\bar{E}$ & $x^{\prime} /|x|^{5}$ & $4 x / 3 r^{4}$ \\
$\bar{F}$ & $x^{\prime}\left(x-x^{\prime}\right) /|x|^{5}$ & $2 / 3 r^{2}$ \\
$\bar{G}$ & $x^{\prime}\left(x-x^{\prime}\right)^{2} /|x|^{5}$ & $2 x / 3 r^{2}$ \\
$\bar{H}$ & $x^{\prime}\left(x-x^{\prime}\right)^{3} /|x|^{5}$ & $-2 x^{2} /\left(1-x^{2}\right)-\frac{8}{3} \ln \left[r^{2} / 4\left(1-x^{2}\right)\right]$ \\
$\bar{I}$ & $\left.x^{\prime}|| x\right|^{7}$ & $16 x / 15 r^{6}$ \\
$\bar{J}$ & $x^{\prime}\left(x-x^{\prime}\right) /|x|^{7}$ & $4 / 15 r^{4}$ \\
$\bar{K}$ & $x^{\prime}\left(x-x^{\prime}\right)^{2} /|x|^{7}$ & $4 x / 15 r^{4}$ \\
$\bar{L}$ & $x^{\prime}\left(x-x^{\prime}\right)^{3} /|x|^{7}$ & $2 / 5 r^{2}$ \\
$\bar{M}$ & $x^{\prime}\left(x-x^{\prime}\right)^{4} /|x|^{7}$ & $2 x / 5 r^{2}$ \\
& &
\end{tabular}

TABLE 2

either $x_{2}$ or $x_{3}$ and hence produce no contribution to (45). To proceed further it is necessary to consider the remaining terms in detail.

The dominant contribution to the remaining integral over $V_{f}$ comes from the region $-1+\epsilon \leqslant x_{1} \leqslant 1-\epsilon, r=O(\epsilon) \ll 1$. Furthermore, it can be shown that the terms in this integral which involve $U_{1}$ and $U_{2}$ explicitly are asymptotically small compared with those involving $f_{1}^{(0)} f_{2}^{(0)}$. Finally, the contribution of the surface integral is smaller than the dominant contribution of the volume integral by $O(1 / \ln \epsilon)$. Hence, neglecting both of these contributions, substituting for $\widetilde{F}_{2}^{(3)}$, $f_{1}^{(0)}$ and $f_{2}^{(0)}$ in the remaining terms from (9), (10) and $(27)$, and finally, integrating over $\phi$, we obtain the result

$$
\begin{aligned}
& G_{3}=\lambda G_{3}^{(1)}+O\left(\lambda^{2}\right) \sim \frac{\lambda \mu U^{*} l^{2} \pi}{16(\ln \epsilon)^{3}} \sin \alpha \cos \alpha \int_{-1+\epsilon}^{1-\epsilon} d x_{1} \int_{R\left(x_{1}\right)}^{c_{1}} r d r \\
& \times\left[\frac{x_{1}^{2}}{r^{2}}\left(\ln \frac{r^{2}}{4\left(1-x_{1}^{2}\right)}\right)^{2}\right]\left(16-96 \epsilon_{1}\right)+O\left(\lambda^{2}\right) .
\end{aligned}
$$

To obtain quantitative results, it is necessary to specify the geometric shape function $R\left(x_{1}\right)$ for the particle. More important, however, are the general qualitative features of (46). First, there is a non-zero torque on the body for all angles of inclination except $\alpha=0$ and $\frac{1}{2} \pi$, for which the particle is oriented with its axis of revolution either vertical or horizontal. For all angles other than 0 and $\frac{1}{2} \pi$, a torque $-G_{3}$ would have to be applied to the particle to keep it from rotating. Hence, in free fall with no externally applied torque, an axisymmetric particle with fore-aft symmetry would rotate towards one of the orientations of zero torque, $\alpha=0$ or $\frac{1}{2} \pi$.

To determine which of these two possible equilibrium orientations is stable, it is necessary to determine the sign of the total coefficient of $\sin \alpha \cos \alpha$ in (46). Since the integrand is positive definite, this may be easily accomplished without specifying any particle geometry function $R\left(x_{1}\right)$. As we have already indicated, it is commonly accepted that $\lambda>0$ and $\lambda \epsilon_{1}<0$ for most viscoelastic fluids 
(cf. Caswell \& Schwarz 1962). In addition, $\ln \epsilon<0$ for $\epsilon \ll 1$. Expressing $G_{3}$ in the general form

$$
G_{3} \sim C \sin \alpha \cos \alpha+O\left(\lambda^{2}\right),
$$

it thus follows that the coefficient

$$
C \equiv \frac{\lambda \mu U^{*} l^{2} \pi}{16(\ln \epsilon)^{3}} \int_{-1+\varepsilon}^{1-\epsilon} d x_{1} \int_{R\left(x_{1}\right)}^{c_{1}} r d r\left[\frac{x_{1}^{2}}{r^{2}}\left(\ln \frac{r^{2}}{4\left(1-x_{1}^{2}\right)}\right)^{2}\right]\left(16-96 \epsilon_{1}\right)
$$

is strictly negative. Referring to figure 1 , it may thus be seen that $\alpha=0$ is stable while $\alpha=\frac{1}{2} \pi$ is unstable to small disorientations. Therefore, in the steady state, an axisymmetric slender particle will fall with its axis of revolution vertical.

So far as we are aware, there exist no published experimental observations which can be compared with the predictions of the present theory. In the appendix of this paper we report on some preliminary experiments which appear to confirm the theoretical prediction of a vertical stable equilibrium orientation for free sedimentation of slender cylinders and cones. A more systematic and careful experimental investigation is currently in progress.

\section{Rotation of a neutrally buoyant particle in a simple shear flow}

\section{Predictions of the theoretical analysis}

We now turn to the angular rotation of a neutrally buoyant, axisymmetric particle with fore-aft symmetry in a simple shearing motion of a second-order fluid. By restricting attention to particles with fore-aft symmetry, we avoid the possibility of shear-induced migration across the flow, so that the particle translates with the local undisturbed velocity of the suspending fluid, as implicitly assumed in (12).

Cox $(1970 a)$ employed slender-body theory to investigate the motion of an axisymmetric particle with fore-aft symmetry in a simple shear flow of a Newtonian suspending fluid. According to Cox's theory, the torque required to produce dimensionless angular rotation rates $\omega_{2}\left(=\omega_{2}^{\prime} / \gamma\right)$ and $\omega_{3}\left(=\omega_{3}^{\prime} / \gamma\right)$ is

$$
\left.\begin{array}{c}
G_{2}^{(0)}=\frac{8}{3} \gamma \pi \mu l^{3}(\ln \epsilon)^{-1}\left(\sin \theta_{1} \cos ^{2} \phi_{1}-\omega_{2}\right), \\
G_{3}^{(0)}=\frac{8}{8} \gamma \pi \mu l^{3}(\ln \epsilon)^{-1}\left(\sin \theta_{1} \cos \theta_{1} \sin \phi_{1} \cos \phi_{1}-\omega_{3}\right),
\end{array}\right\}
$$

in which $l$ is the length of the particle and $\gamma$ the undisturbed shear rate. Hence, in a Newtonian fluid, a particle free from any externally applied couples $\left(G_{2}^{(0)}=G_{3}^{(0)}=0\right)$ will rotate with dimensional angular velocity components

$$
\omega_{2}^{\prime}=\gamma \sin \theta_{1} \cos ^{2} \phi_{1}, \quad \omega_{3}^{\prime}=\gamma \sin \theta_{1} \cos \theta_{1} \sin \phi_{1} \cos \phi_{1} \text {. }
$$

The rates of change of the polar angles $\theta_{1}$ and $\phi_{1}$ follow directly from (49) as

$$
\dot{\phi}_{1}=\gamma \cos ^{2} \phi_{1}, \quad \dot{\theta}_{1}=\gamma \sin \theta_{1} \cos \theta_{1} \sin \phi_{1} \cos \phi_{1} \text {. }
$$

These expressions for $\phi_{1}$ and $\dot{\theta}_{1}$ are the familiar Jeffery (1922) orbit equations for a spheroid of infinite aspect ratio.

In the case of slow motion in a second-order fluid, the torque $G_{i}$ may be expressed as an expansion in the small parameter $\lambda$ :

$$
G_{i}=G_{i}^{(0)}+\lambda G_{i}^{(1)}+O\left(\lambda^{2}\right)
$$


To evaluate the non-Newtonian contribution at $O(\lambda)$, i.e. $G_{i}^{(1)}$, we again use (24) combined with the reciprocal theorem (25). The vector functions $\mathbf{H}$ and $\mathbf{t}$ which appear in the equation (42) [see also (45)] for $G_{i}^{(1)}$ are both evaluated using $\mathbf{u}_{0}=-\mathbf{U}+\mathbf{v}^{(0)}$ with the undisturbed velocity field $-\mathbf{U}$ obtained from (12) and the disturbance field $\mathbf{v}^{(0)}$ calculated using the Stokeslet distribution from (15). As in the previous examples, the surface integrals appearing in the equations for $G_{i}^{(1)}$ are evaluated using the asymptotic forms of the integrand for $r \equiv R\left(x_{1}\right) \ll 1$. Likewise, the volume integrals are dominated by the region very near to the particle but excluding the two end regions $-1+\epsilon \leqslant x_{1} \leqslant 1-\epsilon, r \ll 1$. Since we have restricted our attention to the case of axisymmetric particles with fore--aft symmetry, terms in the integrands which are odd in any of $x_{1}, x_{2}$ (i.e. $\cos \phi)$ or $x_{3}$ (i.e. $\left.\sin \phi\right)$ produce a zero net contribution to the torque $G_{i}^{(1)}$. The detailed evaluations of the integrals are lengthy and of no special interest in themselves, thus we omit them here ${ }^{\dagger}$ and simply report the resulting asymptotic expressions for $G_{2}^{(1)}$ and $G_{3}^{(1)}$ :

$$
\begin{aligned}
& \frac{G_{2}^{(1)}}{\mu \gamma l^{3}} \sim \frac{1}{4 \ln \epsilon}\left[\frac { M _ { 1 } } { \operatorname { l n } \epsilon } \left\{-4 f_{3}^{(0)} A_{22}+f_{1}^{(0)}\left(A_{13}+A_{31}\right)+\epsilon_{1}\left[f_{3}^{(0)}\left(A_{11}-5 A_{22}+2 A_{33}\right)\right.\right.\right. \\
& \left.\left.\left.+2 f_{1}^{(0)}\left(A_{13}+A_{31}\right)-f_{2}^{(0)} A_{23}+f_{1}^{(0)} A_{31}-\frac{d f_{3}^{(0)}}{d t}\right]\right\}-\frac{M_{2}}{512 \pi^{2}(\ln \epsilon)^{2}} f_{1}^{(0)} f_{3}^{(0)}\right], \\
& \frac{G_{3}^{(1)}}{\mu \gamma l^{3}} \sim-\frac{1}{4 \ln \epsilon}\left[\frac { M _ { 1 } } { \operatorname { l n } \epsilon } \left\{-4 f_{2}^{(0)} A_{33}+f_{1}^{(0)}\left(A_{1 \overline{2}}+A_{21}\right)+\epsilon_{1}\left[f_{2}^{(0)}\left(A_{11}+2 A_{22}-5 A_{33}\right)\right.\right.\right. \\
& \left.\left.\left.+2 f_{1}^{(0)}\left(A_{12}+A_{21}\right)+f_{1}^{(0)} A_{21}-f_{3}^{(0)} A_{32}-\frac{d f_{2}^{(0)}}{d t}\right]\right\}+\frac{M_{2}}{512 \pi^{2}(\ln \epsilon)^{2}} f_{1}^{(0)} f_{2}^{(0)}\right] \text {. } \\
& M_{2} \equiv-\int_{-l+R_{0}}^{l-R_{0}} d x_{1} \int_{R\left(x_{1}\right)}^{e_{1}} r d r \mid \frac{12 x_{1}^{2}}{r^{2}}\left(\ln \frac{r^{2}}{4\left(l^{2}-x_{1}^{2}\right)}\right)^{2}+\left(\frac{2 x_{1}^{2}}{r^{2}}+\frac{8 x_{1}^{2}}{r^{2}\left(l^{2}-x_{1}^{2}\right)}\right) \\
& \times \ln \left(\frac{r^{2}}{4\left(l^{2}-x_{1}^{2}\right)}\right) \mid+\left(\hat{n}_{2}+\hat{n}_{3}\right) 8 \pi \mu \int_{-l+R_{0}}^{l-R_{0}} 18 x_{1}^{2} \ln \left(\frac{R^{2}\left(x_{1}\right)}{4\left(l^{2}-x_{1}^{2}\right)}\right) d x_{1} .
\end{aligned}
$$

When evaluated using the definitions for $f_{i}^{(0)}$ and $A_{i j}$ and combined with the Newtonian equation (48), these equations yield explicit relationships between the hydrodynamic torque, particle geometry and the polar orientation angles $\theta_{1}$ and $\phi_{1}$. The general form is $;$

$$
\begin{aligned}
& G_{2} \sim \frac{8}{3} \pi \mu l^{3} \gamma(\ln \epsilon)^{-1}\{\sin \theta \cos ^{2} \phi_{1}-\omega_{2}+K\left[M_{1} h_{1}\left(\theta_{1}, \phi_{1}, \omega_{2}, \omega_{3}\right)\right. \\
&\left.\left.+\left(M_{2} / \ln \epsilon\right) h_{2}\left(\theta_{1}, \phi_{1}, \omega_{2}, \omega_{3}\right)\right]+O\left(K^{2}\right)\right\}
\end{aligned}
$$

and $G_{3} \sim \frac{8}{3} \pi \mu l^{3} \gamma(\ln \epsilon)^{-1}\left\{\sin \theta_{1} \cos \theta_{1} \sin \phi_{1} \cos \phi_{1}-\omega_{3}+K\left[M_{1} h_{3}\left(\theta_{1}, \phi_{1}, \omega_{2}, \omega_{3}\right)\right.\right.$

$$
\left.\left.+\left(M_{2} / \ln \epsilon\right) h_{4}\left(\theta_{1}, \phi_{1}, \omega_{2}, \omega_{3}\right)\right]+O\left(K^{2}\right)\right\}
$$

where

$$
K \equiv 3 \lambda \gamma / 16(\ln \epsilon) \ll 1 \text {. }
$$

$\dagger$ The detailed results of these calculations may be obtained on request from either the author or the JFM Editorial Office.

$\ddagger$ The functions $h_{1}, h_{2}, h_{3}$ and $h_{4}$ may be obtained on request from either the author or the JFM Editorial Office. 
The functions $M_{1}$ and $M_{2}$ depend explicitly on the shape function $R\left(x_{1}\right)$. However, it is sufficient for present purposes to note that

$$
M_{1}>0, M_{2}<0 \text { in the limit } \epsilon \rightarrow 0 .
$$

Now a particle which is free from any externally applied body couples will rotate at a rate $\left(\omega_{2}, \omega_{3}\right)$ such that $G_{2}=G_{3}=0$. Since $K$ appears as a small parameter in the system (54) and (55), we can obtain an asymptotic estimate of the non-Newtonian modification of the dimensionless angular velocities of a freely suspended particle by expanding $\omega_{2}$ and $\omega_{3}$ in the form

$$
\left.\begin{array}{l}
\omega_{2} \sim \omega_{2}^{(0)}+\omega_{2}^{(1)} K+O\left(K^{2}\right), \\
\omega_{3} \sim \omega_{3}^{(0)}+\omega_{3}^{(1)} K+O\left(K^{2}\right) .
\end{array}\right\}
$$

The zeroth-order (dimensional) terms $\omega_{2}^{\prime(0)}$ and $\omega_{3}^{\prime(0)}$ are simply the Newtonian solutions given by (48). Substituting (56) into (54) and (55) and collecting terms $O(K)$, the first correction is easily shown to be

$$
\left.\begin{array}{l}
\omega_{2}^{(1)} \sim M_{1}\left(1+2 \epsilon_{1}\right) \sin ^{3} \theta_{1} \sin \phi_{1} \cos \phi_{1}\left(\sin ^{2} \phi_{1}-\cos ^{2} \phi_{1}\right), \\
\omega_{3}^{(1)} \sim-M_{1}\left(1+2 \epsilon_{1}\right) 2 \sin ^{3} \theta_{1} \cos \theta_{1} \sin ^{2} \phi_{1} \cos ^{2} \phi_{1} .
\end{array}\right\}
$$

Using these expressions, the 'orbit' equations (50) are thus modified to the form

$$
\begin{aligned}
& \dot{\phi}_{1} \sim \gamma \cos ^{2} \phi_{1}+K M_{1}\left(1+2 \epsilon_{1}\right) \gamma \sin ^{2} \theta_{1} \sin \phi_{1} \cos \phi_{1}\left(\sin ^{2} \phi_{1}-\cos ^{2} \phi_{1}\right)+O\left(K^{2}\right), \\
& \dot{\theta}_{1} \sim \gamma \sin \theta_{1} \cos \theta_{1} \sin \phi_{1} \cos \phi_{1} \\
& \quad-2 K M_{1}\left(1+2 \epsilon_{1}\right) \gamma \sin ^{3} \theta_{1} \cos \theta_{1} \sin ^{2} \phi_{1} \cos ^{2} \phi_{1}+O\left(K^{2}\right)
\end{aligned}
$$

For the case of a Newtonian suspending fluid, $K=0$, these equations may be integrated to obtain

$$
\tan \phi_{1}=\gamma t, \quad \tan \theta_{1}=C / \cos \phi_{1},
$$

in which $C$ is the so-called Jeffery orbit constant, which can assume values between 0 and $\infty$ depending upon the initial orientation of the particle. Although a similar inversion of (58) has not been found for non-zero values of $K$, a simple transformation of the equations to the natural $C$, $\tau$ system of Leal \& Hinch (1971) does allow a straightforward illustration of the predicted modifications of the particle motion. Thus we use

$$
\tau=\tan \phi_{1}, \quad C=\tan \theta_{1} \cos \phi_{1}
$$

and transform (58) according to

The result is

$$
\begin{gathered}
d \tau / d t=\sec ^{2} \phi_{1} \dot{\phi}_{1} \\
d C / d \tau=\sec ^{2} \theta_{1} \cos \phi_{1} \dot{\theta}_{1}-\tan \theta_{1} \sin \phi_{1} \dot{\phi}_{1} .
\end{gathered}
$$

$$
\begin{aligned}
& d \tau / d t=\gamma+K M_{1}\left(1+2 \epsilon_{1}\right) \gamma \sin ^{2} \theta_{1} \tan \phi_{1}\left(\sin ^{2} \phi_{1}-\cos ^{2} \phi_{1}\right) \\
& d C / d t=-K M_{1}\left(1+2 \epsilon_{1}\right) \gamma \sin ^{2} \theta_{1} \sin ^{2} \phi_{1} C
\end{aligned}
$$


The first of these equations describes the change of phase of the particle axis with time, whereas the second describes the rate of drift of $C$. The behaviour of the rod depends critically upon the sign and magnitude of the coefficient $K M_{1}\left(1+2 \epsilon_{1}\right) \gamma$ (hereafter denoted as $\beta \gamma$ ). Since $\lambda>0$ and $\epsilon_{1}<0$ for most common viscoelastic fluids, it follows that $K M_{1} \gamma<0$ for $\epsilon \ll 1$. Quantitative rheological information relevant to the coefficient $1+2 \epsilon_{1}$ is more difficult to obtain. Experimental evidence available in 1962 led Caswell \& Schwarz to support the validity of the so-called Weissenberg (1947) hypothesis, according to which the two normal stress components orthogonal to the direction of flow are equal in simple shear, so that

$$
\epsilon_{1}=-\frac{1}{2} .
$$

Clearly, for a true Weissenberg fluid $\beta=0$, and the orbit constant and the rate of rotation through the orbit, as predicted by (61), would be unchanged from the Newtonian case, i.e. $d \tau / d t=\gamma$ and $C=$ constant. However, more recent studies, for example, the experimental measurements of Olabisi \& Williams (1972), Tanner (1970), Wales \& Philippoff (1973), Brindley \& Broadbent (1973) and others, as well as the majority of model studies of particulate suspensions (cf. Hinch \& Leal 1972; Leal \& Hinch 1972; Frankel \& Acrivos 1970; Lin, Peery \& Schowalter 1970) appear to support the proposition that the second normal stress difference is not zero, but small in magnitude compared with the primary normal stress difference and of opposite sign. In this case,

$$
\epsilon_{1}<-\frac{1}{2}
$$

and $K M_{1} \gamma\left(1+2 \epsilon_{1}\right)>0$. According to $(61 a)$, a rod-like particle in a nonNewtonian second-order fluid which satisfies (62) should rotate into the aligned state $\left(\phi_{1}=\frac{1}{2} \pi, \frac{3}{2} \pi\right)$ a little faster than in the corręsponding Newtonian fluid, but come out a little slower. $\dagger$ We shall later show, by taking higher-order corrections to (49), that the rate of change $\phi_{1}$ of the phase angle actually exhibits a stable zero for shear rates above a certain critical value, corresponding to alignment of the particle with a fixed preferred direction. For smaller shear rates, $(61 b)$ indicates that $C$ should decrease towards the equilibrium value $C=0$, with the maximum rate of change of $C$ occurring when $\phi_{1}$ is near $\frac{1}{2} \pi$ or $\frac{3}{2} \pi$.

\section{Comparison with experimental observation and discussion}

Karnis \& Mason (1967), Gauthier et al. (1971) and Bartram \& Mason (1974) have recently made a careful and thorough experimental investigation of the rotational motion of rod-like particles of finite aspect ratio in simple Couette flow using both pseudo-plastic (purely viscous) and viscoelastic suspending fluids. At small shear rates $\gamma$, Karnis \& Mason and Gauthier et al. found that both rods and disks rotated in either type of fluid with a period which differed very little from the Jeffery-orbit value for a rod or disk of the same finite aspect ratio in

$\dagger$ Strictly speaking, the long slender rods $(r=\infty)$ which we have considered do not cross the aligned state at all as we have noted earlier. However, for purposes of the present discussion, we shall assume that any particle which arrives at $\phi_{1}=\frac{1}{2} \pi$ or $\frac{3}{2} \pi$ with $\theta_{1}=\frac{1}{2} \pi$ will cross as a result of the small torque $O\left(\epsilon^{2}\right)$ which exists there (Cox $\left.1970 b\right)$, but which is outside the scope of the present slender-body analysis. 
a Newtonian fluid. In a Newtonian fluid the particles rotate about fixed orbits $C$. However, at moderate to small shear rates in both pseudo-plastic and viscoelastic fluids, Gauthier et al. and Bartram \& Mason found that a reasonably rapid drift to $C=0$ occurred in every case for rod-like particles, with the largest changes in $C$ occurring when $\phi_{1}$ was within $\pm 10^{\circ}$ of $\frac{1}{2} \pi$ or $\frac{3}{2} \pi$. In addition, for moderate $\gamma$, Bartram \& Mason reported periods of rotation for both rods and disks in a $25 \%$ polyacrylamide (PAA) in water solution which were markedly greater than those predicted by the Jeffery theory. Finally, above some critical shear rate, Bartram \& Mason reported that sufficiently long rods in viscoelastic media appeared to align permanently with $\phi_{1}$ and $\theta_{1}$ both very near to $\frac{1}{2} \pi$. For these supercritical shear rates, orbit drift to $C=0$ was also sometimes observed, but only after the 'permanently' aligned particle was subjected to an extra disturbance such as that caused by interaction with a second nearby particle. Qualitatively, these observations of orbit drift and the predictions of $(61 b)$, as outlined in the previous section, show a remarkable degree of correspondence. The only previous theoretical analysis (Saffman 1956) not only fails to predict any of the detailed variations in the rate of change of $C$ with orientation $\left(\phi_{1}\right)$, but also produces no criteria to determine whether $C$ should increase or decrease in time.

A more quantitative comparison between theory and experiment is difficult, because the experimental particles have a finite axis ratio and thus rotate periodically through $\phi_{1}=0-2 \pi$, whereas the particles of infinite axis ratio considered by the theory align with no further rotation near $\phi_{1} \sim \frac{1}{2} \pi$ or $\frac{3}{2} \pi$ even in the Newtonian limit. In order to achieve periodic rotation (58) must be modified to account for the small but non-zero torque acting on the particle when $\phi_{1} \simeq \frac{1}{2} \pi(2 n+1)$ (where $n$ is any arbitrary non-negative integer). To do this in a rigorous way, both the basic Newtonian and non-Newtonian slender-body solutions would have to be modified to account for higher-order, $O\left(\epsilon^{2}\right)$, effects. The Newtonian result is easily obtained from the exact Jeffery-orbit equations

and

$$
\begin{gathered}
\dot{\phi}_{1}=\frac{\gamma}{r^{2}+1}\left(r^{2} \cos ^{2} \phi_{1}+\sin ^{2} \phi_{1}\right) \\
\dot{\theta}_{1}=\frac{\gamma\left(r^{2}-1\right)}{r^{2}+1} \sin \theta_{1} \cos \theta_{1} \sin \phi_{1} \cos \phi_{1} .
\end{gathered}
$$

Letting $r \rightarrow \infty$, these reduce to the slender-body equations (50) for a Newtonian fluid, except for $\phi_{1} \sim \frac{1}{2} \pi(2 n+1)$, when

$$
\phi_{1} \sim \gamma / r^{2}
$$

This small $O\left(r^{-2}\right)$ term is precisely the higher-order correction term which would be obtained, for a Newtonian fluid, from the slender-body analysis which we have referred to above, and which is responsible for the slow rotation of a real particle in a Newtonian fluid through the aligned positions $\phi_{1} \sim \frac{1}{2} \pi(2 n+1)$. A useful and uniformly valid first approximation to the orbit equations in the Newtonian case may thus be obtained simply by combining the first-order slender-body equations (50) with the expression (64) in the form

$$
\begin{aligned}
& \dot{\phi}_{1} \sim \gamma\left(\cos ^{2} \phi_{1}+r^{-2}\right), \\
& \dot{\theta}_{1} \sim \gamma \sin \theta_{1} \cos \theta_{1} \sin \phi_{1} \cos \phi_{1} .
\end{aligned}
$$


As a test of the accuracy of these equations for $r=16 \cdot 1$ (the value used by Mason and co-workers in many of their non-Newtonian experiments) we have integrated them numerically using a simple Runga-Kutta scheme, and compared the results with similar calculations based on the exact equations (63). The detailed orbit shapes are nearly identical. In addition, the orbit period $T^{\prime}$ for these approximate equations,

$$
T^{\prime}=\frac{2 \pi}{\gamma} \frac{r^{2}}{\left(r^{2}+1\right)^{\frac{1}{2}}} \sim \frac{2 \pi}{\gamma}\left(r-\frac{1}{2} r^{-1}+\ldots\right),
$$

compares favourably for $r=16 \cdot 1$ with the exact theoretical result

$$
T=(2 \pi / \gamma)\left(r+r^{-1}\right) \text {. }
$$

In the case of a second-order suspending fluid, an additional non-Newtonian correction to the orbit equations (58) would be expected at $O\left(\lambda \epsilon^{2}\right)$ as we have indicated above. However, since the exact orbit equations are not known in this case, we at present have no practical way of estimating this additional correction term. In spite of this, we have felt it useful, in qualitative terms, to proceed with the numerical and analytical evaluation of (58) for various values of $\beta$, using (64) as an approximation for $\phi_{1}$ when $\phi_{1} \sim \frac{1}{2} \pi(2 n+1)$, i.e.

$$
\begin{aligned}
& \dot{\phi}_{1} \sim \gamma\left(\cos ^{2} \phi_{1}+r^{-2}\right)+\beta \gamma \sin ^{2} \theta_{1} \sin \phi_{1} \cos \phi_{1}\left(\sin ^{2} \phi_{1}-\cos ^{2} \phi_{1}\right), \\
& \dot{\theta}_{1} \sim \gamma \sin \theta_{1} \cos \theta_{1} \sin \phi_{1} \cos \phi_{1}-2 \gamma \beta \sin ^{3} \theta_{1} \cos \theta_{1} \sin ^{2} \phi_{1} \cos ^{2} \phi_{1} .
\end{aligned}
$$

In the remainder of this section, we describe the results of these calculations.

Two features of the particle motion in the second-order fluid are of special interest: the gradual drift through Jeffery orbits and the variation in the phase about the 'orbits' with time, particularly the existence of a permanent or nearly permanent stable orientation for large shear rates $\gamma$.

We begin by examining ( $66 a$ ) for the possible existence of an orientation of permanent alignment for large shear rates. Thus we put $\dot{\phi}=0$ and search for real roots of the resulting transcendental equation in $\phi_{1}$, for fixed values of $\theta_{1} \cdot \dagger$ The problem is simplified by anticipating from Bartram \& Mason's experiments that these roots will be near to $\phi_{1}=\frac{1}{2} \pi, \frac{3}{2} \pi$. Hence, introducing the simple transformation $\phi_{1}=\frac{1}{2} \pi+\phi^{\prime}$ and noting that $\phi^{\prime} \ll 1,(66 a)$ with $\dot{\phi}_{1}=0$ may be approximated by the simple quadratic form

which has roots

$$
0=\gamma\left[\phi^{\prime 2}-\beta \sin ^{2} \theta_{1}\left(\phi^{\prime}+O\left(\phi^{\prime 3}\right)\right)+r^{-2}+O\left(\phi^{\prime 4}\right)\right]
$$

$$
\phi^{\prime}=\frac{1}{2}\left[\beta \sin ^{2} \theta_{1} \pm\left(\beta^{2} \sin ^{4} \theta_{1}-4 / r^{2}\right)^{\frac{1}{2}}\right] .
$$

The condition for the existence of physically realizable stationary points is that these roots be real, namely

$$
\beta \sin ^{2} \theta_{1} \geqslant 2 / r \text {. }
$$

When this condition is satisfied, both roots are positive and it is easily shown that the smaller of the two is a stable equilibrium point of $(66 a)$. The physical signif-

$\uparrow$ Although the zeros of $\dot{\theta}_{1}$ do not coincide precisely with those of $\dot{\phi}_{1}$, we shall show that the stable zero of $\dot{\phi}_{1}$ provides an adequate condition for permanent alignment. 
cance of (69) is most easily shown by substituting the definition $K M_{1}\left(1+2 \epsilon_{1}\right)$ for $\beta$, and evaluating $M_{1}$ using $R\left(x_{1}\right)=\epsilon\left(1-x_{1}^{2}\right)^{\frac{1}{2}}$ to give

$$
\gamma^{2} \geqslant-\frac{16}{r \sin ^{2} \theta_{1}}\left[\frac{\mu}{\Phi_{3}+2 \Phi_{2}}\right]
$$

The minimum or critical value of $\gamma$, for $\dot{\phi}=0$, occurs for $\theta_{1}=\frac{1}{2} \pi$,

$$
\gamma_{\mathrm{er}}^{2}=-\frac{16}{r}\left[\frac{\mu}{\Phi_{3}+2 \Phi_{2}}\right]
$$

and the corresponding angle of alignment $\phi_{\mathrm{cr}}^{\prime}$ is

$$
\phi_{\mathrm{cr}}^{\prime}=1 / r \text {. }
$$

In relating the prediction of a critical shear rate for $\dot{\phi}_{1}=0$ to the experimental observation of 'permanent' alignment by Bartram \& Mason (1974), it is critical to note, first, that

$$
\dot{\theta}_{1} \sim\left(c_{1} \sin \theta_{1} \cos \theta_{1}\right) r^{-\frac{3}{2}}
$$

for $\gamma=\gamma_{\mathrm{cr}}$, and second, that for $r \gg 1$ nearly all of the possible Newtonian orbits pass within $O\left(r^{-1}\right)$ of the wholly aligned state $\left(\theta_{1}=\phi_{1}=\frac{1}{2} \pi\right)$. In the latter region

$$
\dot{\theta}_{1} \sim O\left(r^{-\frac{5}{2}}\right) \dagger
$$

and the experimental observation of permanent alignment for sufficiently large shear rates is thus qualitatively indistinguishable from the predictions of the present theory. A more detailed comparison with experimental results will be presented at the end of this section.

A definitive explanation of the further observation of Bartram \& Mason that particle interactions or other disturbances to the flow could cause the particles to resume their angular motion and drift into the orbit $C=0$ is, of course, not possible in the context of the present theory. Nevertheless, a qualitative understanding of the phenomenon appears possible. Since the particles remain more or less permanently aligned, disturbances associated with particle interactions, or other random phenomena such as rotary Brownian motion, obviously have their greatest influence on particles which are in the aligned state. A small random rotation away from the aligned state may either transport the particle to a new orbit which also passes through a position of stable alignment or, if the rotation is near to the $x, z$ plane of figure 2 , onto an orbit which crosses the $x, z$ plane with $\theta_{1}$ outside the domain of stable orientations. The role of the disturbance rotation, which Bartram \& Mason (1974) have found to be critical to the initiation of orbit drift at large shear rates, is simply to provide a mechanism for transfer into orbits (small $C$ ) which 'miss' the aligned state. When this happens, the subsequent orbit decay to $C=0$ takes place in the manner described by $(61 b)$. One implication of this idea and the $\sin ^{2} \theta$ dependence of (68) and (69) which could be checked experimentally is that an increase of $\gamma$ above the critical value should lead to a weak smearing of the set of aligned orientations over an increasing portion of the $x, z$ plane (i.e. a greater range of $\theta_{1}$ ). To date, there are no experimental results of which we are aware to either confirm or refute this prediction.

$$
\dagger \text { Note, } \dot{\theta}_{1} \equiv 0 \text { for } \theta_{1} \equiv \frac{1}{2} \pi \text {. }
$$




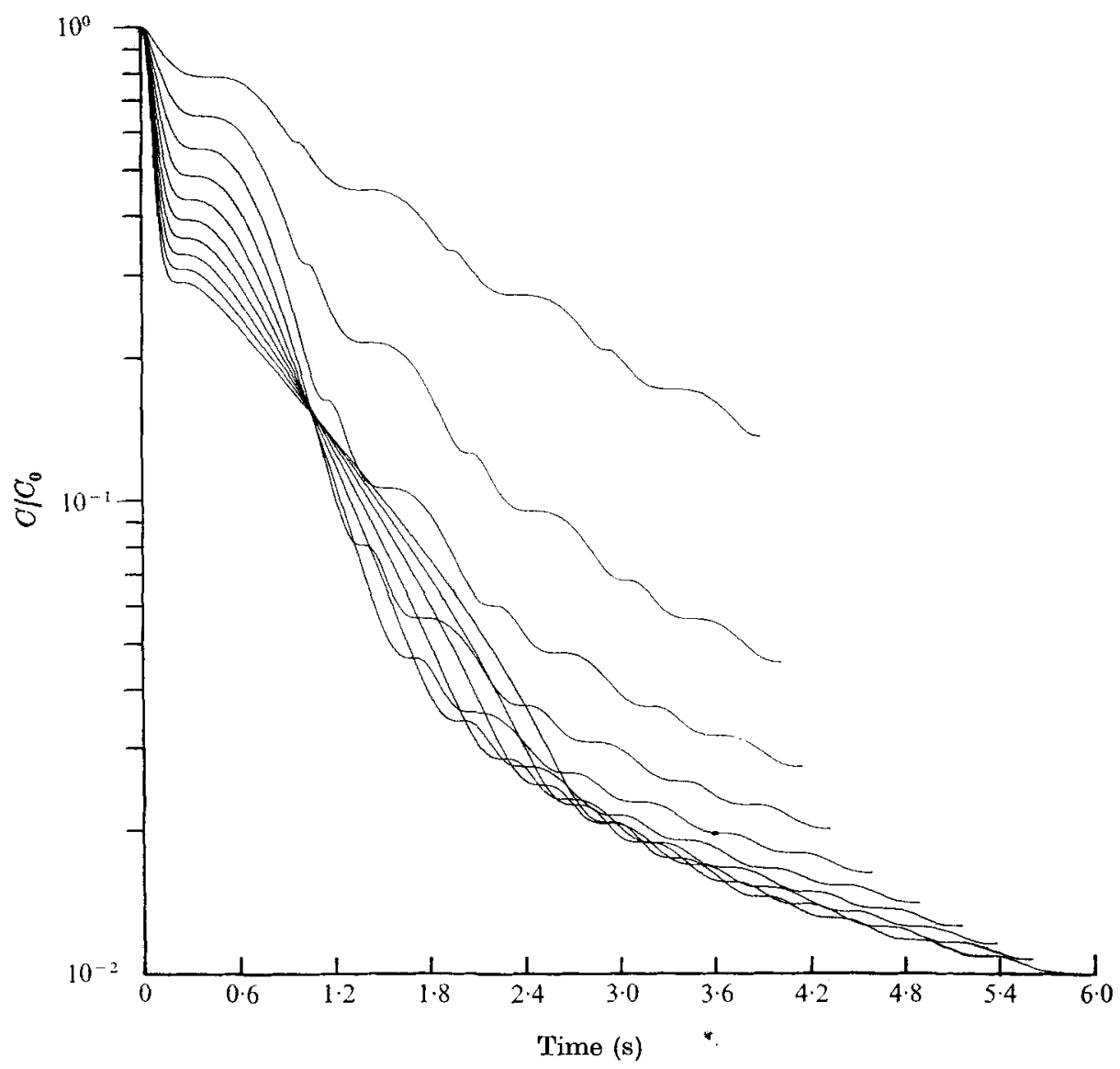

Figure 4. Change in the orbit constant $C$ as a function of time for $\beta$ ranging from +0.025 to +0.25 in increments of $+0.025 ; C_{0}=1.8, r=16.1$ and $\gamma=0.53 \mathrm{~s}^{-1}$.

When $\gamma<\gamma_{\mathrm{cr}}$, or if $C$ is sufficiently small, the particle motion is periodic and characterized experimentally by the phenomenon of orbit drift. We begin our study of this regime of smaller shear rates by considering the variations in particle dynamics as a function of $\beta$ for $\gamma=0.53 \mathrm{~s}^{-1}$ and $r=16 \cdot 1$. These values for $\gamma$ and $r$ were chosen to provide direct comparison with the available experimental results of Gauthier et al. (1971). Equations (66) were integrated numerically to determine $\theta_{1}$ and $\phi_{1}$ as functions of time for various values of $\beta$. The orbit constant corresponding to a given point $\left(\theta_{1}, \phi_{1}\right)$ was then calculated using the definition

$$
C \equiv \tan \theta_{1} \cos \phi_{1}\left(1+r^{-2} \tan ^{2} \phi_{1}\right)^{\frac{1}{2}}
$$

relevant to particles of finite aspect ratio. The term proportional to $r^{-2}$ is, of course, negligible for $r \gg 1$ provided that $\phi_{1}$ is not too near $\frac{1}{2} \pi(2 n+1)$. In this case, $C \sim \tan \theta_{1} \cos \phi_{1}$. However, initial calculations with $\beta \equiv 0$ showed $C$ to vary widely when this limiting expression was used, but to remain constant to within $0.1 \%$ over three complete orbits ( $\phi_{1}$ increasing from 0 to $6 \pi$ ) with (72). Gauthier et al. (1971) have plotted experimentally determined values for log $\left(C / C_{0}\right)$ 


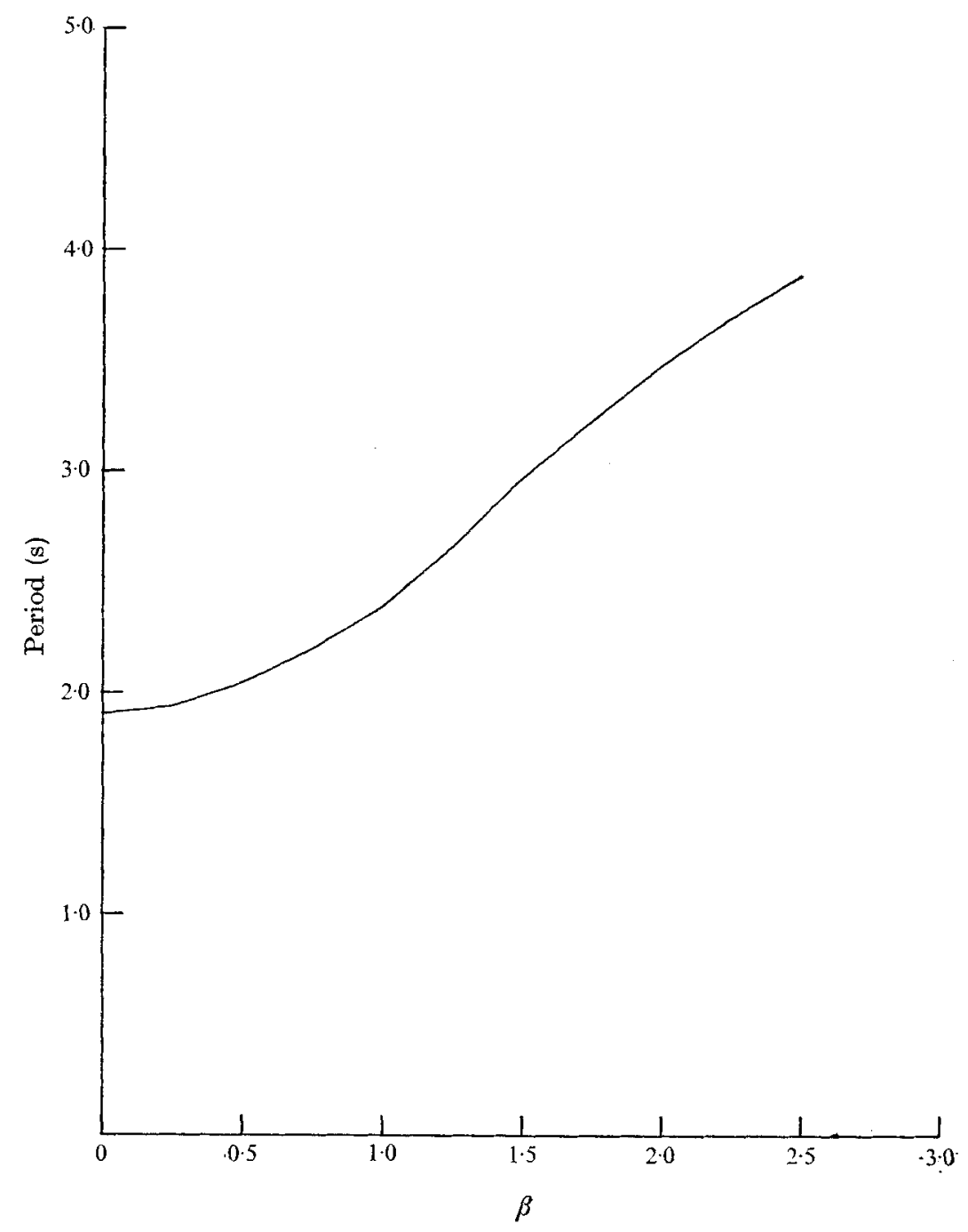

Figure 5. Period of rotation $\left(\phi_{1}=0-2 \pi\right)$ as a function of $\beta$. $C_{0}=1.8, r=16.1$ and $\gamma=0.53 \mathrm{~s}^{-1}$.

as a function of time for $\gamma=0.53 \mathrm{~s}^{-1},\left.C_{0} \equiv C\right|_{t=0}=1.8$ and $r=16.1$ for a $3 \%$ polyacrylamide-water solution. The data fell approximately on a straight line over a period of about two complete rotations (in $\left.\phi_{1}\right)+$ Since the particle spends the majority of each orbit period with $\phi_{1} \sim \frac{1}{2} \pi(2 n+1)$, this result implies that the rate of orbit drift is independent of $\phi_{1}$ for $\phi_{1}$ near the aligned state. We have calculated $C$ as a function of time from the present theory, as described above, for values of $\beta$ ranging from 0 to +0.25 in increments of 0.025 , and $C_{0}, r$ and $\gamma$ equal to the experimental values of Gauthier et al. (1971). The results are shown in figure 4 as a semi-log plot of $C / C_{0} v s$. time. In each case, the curve was

$\dagger$ Professor Mason has indicated, in private correspondence, that the time scale of figure 9 in Gauthier et al. (1971) should be doubled. 


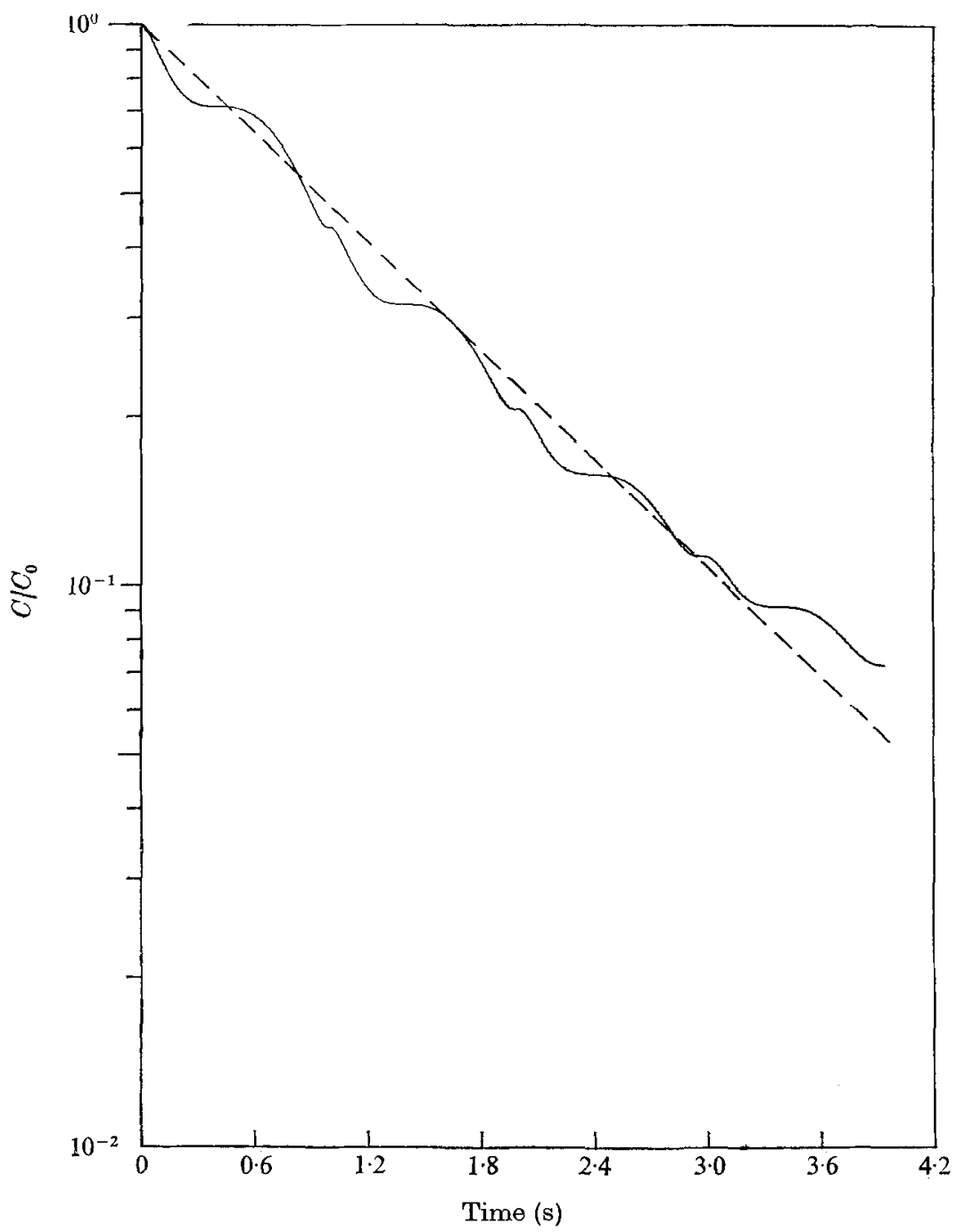

Figure 6. A comparison of the measured and calculated rates of change of the orbit constant for $\beta=0.037 ; C_{0}=1.8, \gamma=16.1$ and $\gamma=0.53 \mathrm{~s}^{-1}$. - . present theory; - - - experiment, Gauthier et al. (1971).

plotted over two complete rotations in $\phi_{1}$ (from 0 to $4 \pi$ ). For the smallest value of $\beta(\equiv+0.025)$ the decrease in $C / C_{0}$ with time is nearly linear (on this semi-logarithmic plot) over two rotations (orbits), though a smaller-scale wiggle superimposed on the main linear variation is clearly evident. As implied above, this small-scale structure is primarily a reflexion of different rates of drift for different values of $\phi_{1}$ near $\frac{1}{2} \pi(2 n+1)$. In particular, careful study of the curves of figure 4 shows that the secondary wave pattern is a result of more rapid orbit drift in the post-aligned orientations $\left(\phi_{1}\right.$ still approximately $\left.\frac{1}{2} \pi\right)$ than in the approaching angles. For larger values of $\beta$ the wiggles in the curves are magnified, reflecting an increased bias toward post-alignment drift and there is a marked fall-off in the 


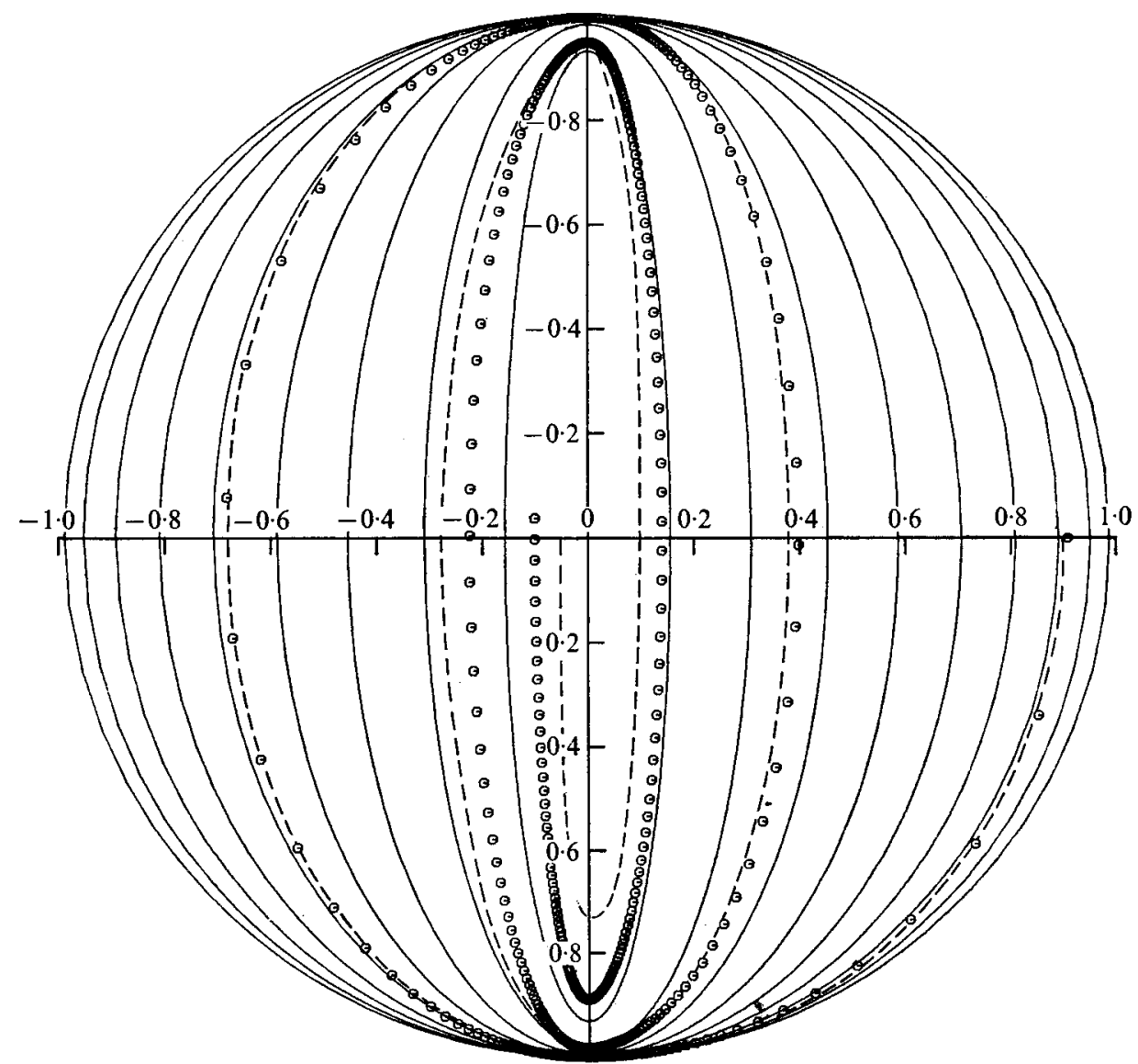

FrGURE 7. A comparison of the detailed particle rotation as obtained by the projection of the end of the particle onto the plane of the shear flow $(x, y) ; C_{0} \sim 2 \cdot 176, \beta=0 \cdot 037$, $r=16.1, \gamma=0.53 \mathrm{~s}^{-1}$. — , Jeffery orbits as calculated from (63); - - , experimentally measured orbits in $2.5 \%$ PAA, Gauthier et al. (1971); $\Theta$, theoretical orbits as calculated from (66). The points are equally spaced in time with an increment of $0 \cdot 75 \mathrm{~s}$ between successive points.

rate of decrease of $C / C_{0}$ for the larger times (or equivalently, for the smaller values of $C$ ). Also noteworthy is the obvious fact that the dependence on $\beta$ becomes weaker as $\beta$ is increased, particularly for large times (small $C$ ), where all the curves for $\beta$ between +0.15 and +0.25 are very close together. Finally, a comparison of the various curves for different values of $\beta$ indicates a clear increase in the period of rotation for increasing $\beta$. This last feature is explicitly illustrated in figure 5, where we have plotted the elapsed time for an increase of $\phi_{1}$ from 0 to $2 \pi$ as a function of $\beta$ for $C_{0}=1 \cdot 8, \gamma=0.53 \mathrm{~s}^{-1}$ and $r=16 \cdot 1$. It should be noted, however, that the change in period with increasing $\beta$ is a strong function of $C_{0}$, being nearly independent of $\beta$ for $C_{0}<0 \cdot 18$.

The value of $\beta$ which provides the best match between the experimental and theoretical values of $\log \left(C / C_{0}\right)$ as a function of time is $\beta=+0.037$. The correspond- 
ence between experiment and theory for this value of $\beta$ is illustrated in figure 6 . The only additional quantitative data presented by Gauthier et al. (1971) are for the projection of the end of the axis of revolution onto the $x, y$ plane. Unfortunately, however, the precise projection is quite sensitive to the initial value of $C$, which is not stated. In order to provide an approximate comparison, we have estimated $C_{0} \sim 2.176$ at the second crossing of $\phi_{1}=0$ from their figure $7(b)$. The $x, y$ projection of the particle axis as it rotates through $\phi_{1}$ from 0 to $5 \pi$ was calculated using (66) for $\gamma=0.53 \mathrm{~s}^{-1}, r=16 \cdot 1, C_{0}=2.176$ and $\beta=+0.037$ and the results superimposed onto a plot of the various Jeffery orbits and available experimental data (figure 7). Most clearly evident, on comparing the calculated Newtonian and non-Newtonian orbits, is the fact that the major deviations between the two occur for $\phi_{1} \sim \frac{1}{2} \pi(2 n+1)$. Hence, as we have indicated earlier, little orbit drift occurs except near the aligned orientations, $\phi_{1}=\frac{1}{2} \pi, \frac{3}{2} \pi$. Furthermore, the skewness in the rate of orbit drift between preand post-alignment values of $\phi_{1}$ is also evident from the relatively small deviations from the Newtonian orbits for $\phi_{1}<\frac{1}{2} \pi, \frac{3}{2} \pi$ (but near these values), and the large deviations for $\phi_{1}>\frac{1}{2} \pi, \frac{3}{2} \pi$. Of greatest significance, however, is the excellent qualitative agreement with the experimental projections of Gauthier et al. (1971). Although the small scale of their figure $7(b)$ makes comparison difficult, we have attempted to transpose their data onto our figure 7 for this purpose. It is our belief that the small apparent differences are a result of limited experimental accuracy, possible error in the estimated value for $C_{0}$, and the fact that no systematic attempt was made to improve on the estimate $\beta=0.037$ from figure 6. In particular, the approximation inherent in the use of (64) does not appear to be a serious limitation for the theory.

As a final point concerned with orbit drift, we add an addendum to a remark of Karnis \& Mason (1967), who suggested that the rate of change of the orbit constant might prove to be a sensitive measure of 'viscoelasticity'. The present theoretical development does, in fact, indicate that the rate of orbit drift should be directly proportional to the parameter $\lambda\left(1+2 \epsilon_{1}\right)$ of the Rivlin-Erickson constitutive model provided that the local fluid motion is rheologically slow as we have assumed. In addition, the direction of change, i.e. whether $C$ increases or decreases in time, depends upon whether $1+2 \epsilon_{1}$ is positive or negative. Practically speaking, the parameter $\Phi_{2}$ (proportional to $\epsilon_{1} \lambda$ ) is relatively easily measured. Measurement of the parameter $\Phi_{3}$, corresponding to the second normal stress difference in simple shear flow, is much more difficult as we have already noted. The present theory suggests that accurate observations of the motion of single particles in simple shear flow, particularly the rate and direction of orbit drift, can provide a sensitive measure of the second normal stress difference parameter $\Phi_{3}$, when coupled with an independent determination of $\Phi_{2}$. We have noted above that $K M_{1}\left(1+2 \varepsilon_{1}\right)=0.037$ appeared to provide the best fit of experimental data on orbit drift for a rod-like particle of aspect ratio 16.1 in a $3 \%$ by weight polyacrylamide-water solution. For this system, Gauthier et al. (1971) made measurements of the shear dependence of viscosity and primary normal stress difference. Later, Bartram \& Mason (1974) repeated these measurements using an orthogonal rheometer and a $2.5 \%$ PAA solution. Substituting 
the definitions of $K, M_{1}, \gamma$ and $\epsilon_{1}$ (assuming $\left.R\left(x_{1}\right)=\epsilon\left(1-x_{1}^{2}\right)^{\frac{1}{2}}\right)$ into the estimate $K M_{1}\left(1+2 \epsilon_{1}\right)=0.037$ and rearranging gives

$$
\Phi_{3}=-0 \cdot 037(8 \mu) / \gamma^{2}-2 \Phi_{2}
$$

In order to estimate $\Phi_{3}$, it is necessary to use the rheological data of Gauthier et al. (1971) and Bartram \& Mason (1974) to obtain $\mu$ and $\Phi_{2}$. By definition, for the second-order fluid limit,

$$
\begin{gathered}
\mu \equiv \lim _{\gamma \rightarrow 0}\left(\sigma_{12} / \gamma\right), \\
\Phi_{2} \equiv-\lim _{\gamma \rightarrow 0} \frac{\sigma_{11}-\sigma_{22}}{2 \gamma^{2}}, \quad \Phi_{3} \equiv \lim _{\gamma \rightarrow 0} \frac{\sigma_{11}-\sigma_{33}}{\gamma^{2}} .
\end{gathered}
$$

Unfortunately, although the measurements of Gauthier et al. (1971) and Bartram \& Mason (1974) of viscosity vs. shear rate agree, neither extend to sufficiently low shear rates to allow estimation of the lower limiting viscosity $\mu$. In addition, not only do the normal stress measurements also not extend to sufficiently low shear rates, but the presumably more accurate data of Bartram \& Mason (1974) are larger than those of Gauthier $\mathrm{et} \mathrm{al}$. by a factor of 20-60 over the range of shear rates used. Since Bartram \& Mason's (1974) measurements were made for $2.5 \%$ PAA, it is difficult to estimate appropriately corrected primary normal stress values for the $3 \%$ solution. However, as a preliminary test of the basic idea of using orbit drift measurements to calculate $\Phi_{3}$, we use estimated values of $\mu$ and $\Phi_{2}$ at $\gamma=0.53 \mathrm{~s}^{-1}$ (the shear rate in the drift experiments). Accordingly, $\mu \sim 65 \mathrm{P}$, while extrapolation of the ratio of the new $2.5 \%$ data and the original $3 \%$ data to $\gamma=0.53 \mathrm{~s}^{-1}$ yields the estimate $\Phi_{2} \sim-195$ dyne $\mathrm{s}^{2} \mathrm{~cm}^{-2}$. Hence, from (72), the corresponding 'best-fit' value of $\Phi_{3}$ is 321 dyne $\mathrm{s}^{2} \mathrm{~cm}^{-2}$. Thus $\epsilon_{1} \sim-0 \cdot 6$. This value corresponds to the second normal stress difference in simple shear flow being of opposite sign and having a magnitude equal to approximately $17 \%$ of the primary normal stress difference. Similar values have been reported by most recent investigators, and there is now general agreement that second normal stress differences are smaller and opposite in sign compared with primary normal stress differences in simple shear flow of viscoelastic fluids. Presumably, better estimates of $\Phi_{2}$ and $\mu$ would lead to more definite results for $\Phi_{3}$ (and hence $\epsilon_{1}$ ). Unfortunately, we know of no other measurement of $\epsilon_{1}$ for this specific system ( $3 \%$ by weight polyacrylamide) which can be compared with our estimate.

Finally, we return briefly to the phenomenon of permanent alignment for large shear rates, as described by (67)-(71). Clearly, careful measurement of $\gamma_{\mathrm{cr}}$, the axis ratio $r$ and the shear viscosity and primary normal stress difference provides an alternative measurement of $\Phi_{3}$ through (70). Unfortunately, though Bartram \& Mason demonstrated the existence of a critical shear rate for permanent alignment of rod-like particles in a viscoelastic fluid, the maximum (effective) particle axis ratio which they employed was $r=6 \cdot 27$, too small to expect quantitative agreement with the present slender-body theory. Nevertheless, we have felt it useful to use the values of $\mu, \Phi_{2}$ and $\Phi_{3}$ obtained above to estimate $\gamma_{\mathrm{cr}}$ for comparison with the experimental value. Hence, substituting $r=6 \cdot 27, \mu=65, \Phi_{3}=321$ and $\Phi_{2}=-195$ into (70), we obtain, for $\theta=\frac{1}{2} \pi$,

$$
\gamma_{\mathrm{cr}}=1.55 \mathrm{~s}^{-1} \text {. }
$$


The value observed by Bartram \& Mason (1974) was $5 \mathrm{~s}^{-1}$. Although the agreement is not particularly impressive, the rheological data we employed were for $\gamma=0.5 \mathrm{~s}^{-1}$, considerably smaller than the calculated (and measured) critical shear rate. Furthermore, the theory is for a second-order fluid and a highly elongated particle while the measurements were taken well beyond the secondorder fluid regime using a particle with $r=6 \cdot 27$. In view of these facts, the lack of quantitative agreement is not surprising. Again, a careful and comprehensive set of experiments would be useful in verifying the various predictions of the theory.

This work was supported by the National Science Foundation under grant GK-35468. The author wishes to acknowledge helpful discussions with $\mathbf{M r}$ Bosco Ho and Dr E. J. Hinch on various aspects of this work. The Separan AP30 was donated by the Dow Chemical Company.

\title{
Appendix. A preliminary experimental investigation of the motion of slender particles in Separan-AP30
}

\author{
By L. G. Leal and E. Zana \\ Chemical Engineering, California Institute of Technology, Pasadena
}

As we have indicated in the body of the paper, some preliminary qualitative experiments were performed in order to provide a partial verification of the predictions of the present theory. In view of the extensive studies in shear flow by Mason and co-workers the present experimental studies have been focused entirely on the translation of slender axisymmetric particles through a quiescent fluid.

For this work we used two circular cylindrical particles with rounded ends, lengths 0.704 and $0.724 \mathrm{in}$., and axis ratios of 28 and 66 , as well as a circular cone with a length of $0.681 \mathrm{in}$. and a maximum diameter of $0.053 \mathrm{in}$. The shorter cylinder and the cone were machined from block aluminium, while the longer cylinder was cut from a piece of stainless-steel wire. The experiments performed were limited to sedimentation of a single particle in a Plexiglas tank which was $18 \mathrm{in}$. in height and 6 in. square in cross-section. The fluids used were $99.5 \%$ glycerine (Newtonian), with a viscosity of about $15 \mathrm{P}$, and a $0.5 \%$ solution of Dow's Separan AP30, which has a similar value for its low shear limiting viscosity but is fully viscoelastic. Typical shear viscosity and primary normal stress data for Separan have been presented in a number of previous publications (cf. Skoog, Leal \& Acrivos 1971; Bruce \& Schwarz 1969) and so will not be repeated here. The sedimentation experiments were carried out by introducing the particles into the top of the tank with some predetermined orientation, and then observing their motion and orientation over a span at the midlevel of the tank using a strobatac light source in conjunction with a Graflex Crown Reflex Camera to produce multiple-image photographs in which the successive images of the particle were separated by a known increment of time. 
It was anticipated that wall-particle interactions might play a critical role in determining the particle motion in a tank of this size. Indeed, the very recent theoretical and experimental work of de Mestre (1973) for a Newtonian suspending fluid had already suggested that the terminal velocity could be measurably affected for a ratio of particle length to tank width of the order of 0.05 or less. In addition, de Mestre indicated that particle-wall interactions may cause the orientational behaviour of the particle to differ markedly from that expected in an unbounded body of the same Newtonian fluid. For this reason, experiments were carried out in both Newtonian and non-Newtonian fluids in order that the former might provide a frame of reference from which the explicitly nonNewtonian contributions to the particle's motion could be clearly distinguished.

In the present work, we have attempted to verify only two qualitative predictions of the theory: first, that particles with fore-aft symmetry fall with a preferred vertical orientation in the steady state, and second, that cone-shaped particles tend to fall more slowly with the blunt end leading than with the pointed end leading.

We first consider the results for particle orientation in a Newtonian fluid. In this case, we have found that the orientation remains fixed provided that the particle does not drift too close to the side walls of the tank. A typical multiimage photograph taken at the midlevel of the tank is shown as figure 8 (plate 1), and illustrates the essential lack of change of particle orientation. The orientation shown is, in fact, within a few degrees of that initially imposed at the top of the tank. In addition, it may be noted that the direction of fall is not vertical, but instead shows a significant sideways component across the plane of motion. This behaviour is expected as a result of the familiar $2: 1$ ratio of drag for motion perpendicular or parallel to the axis of a very slender particle in a Newtonian fluid. A simple calculation based on this ratio shows that

$$
\tan \alpha=\frac{1}{2} \cot \delta,
$$

in which $\delta$ is the inclination of the particle axis and $\alpha+\delta$ is the inclination of the path of motion, both measured from the horizontal plane. We have measured $\alpha$ and $\delta$ directly from the photographs for a number of the Newtonian experiments. The results are shown compared with the theoretical expression (73) in figure 9. The small but consistent deviations between theory and experiment are caused by the finite aspect ratio of the particles, which would be expected to produce a drag ratio smaller than $2: 1$. Hence, although the sideways drift obviously holds the potential for complicated particle-wall interactions as the particles drift towards a wall, the motion actually observed is indistinguishable on the scale of the present experiments from that for an unbounded body of fluid provided only that the particle is not too near a wall.

The particle motion in Separan AP30 is markedly different, as may be seen by comparing the multi-image photograph of figure 10 (plate 1) for Separan with that of figure 8 for the Newtonian glycerine solution. In particular, the same particles which sedimented with constant orientation through glycerine were found in every case to rotate rapidly from their initial orientation to a vertical orientation when sedimenting through the Separan-water solution. It should be 


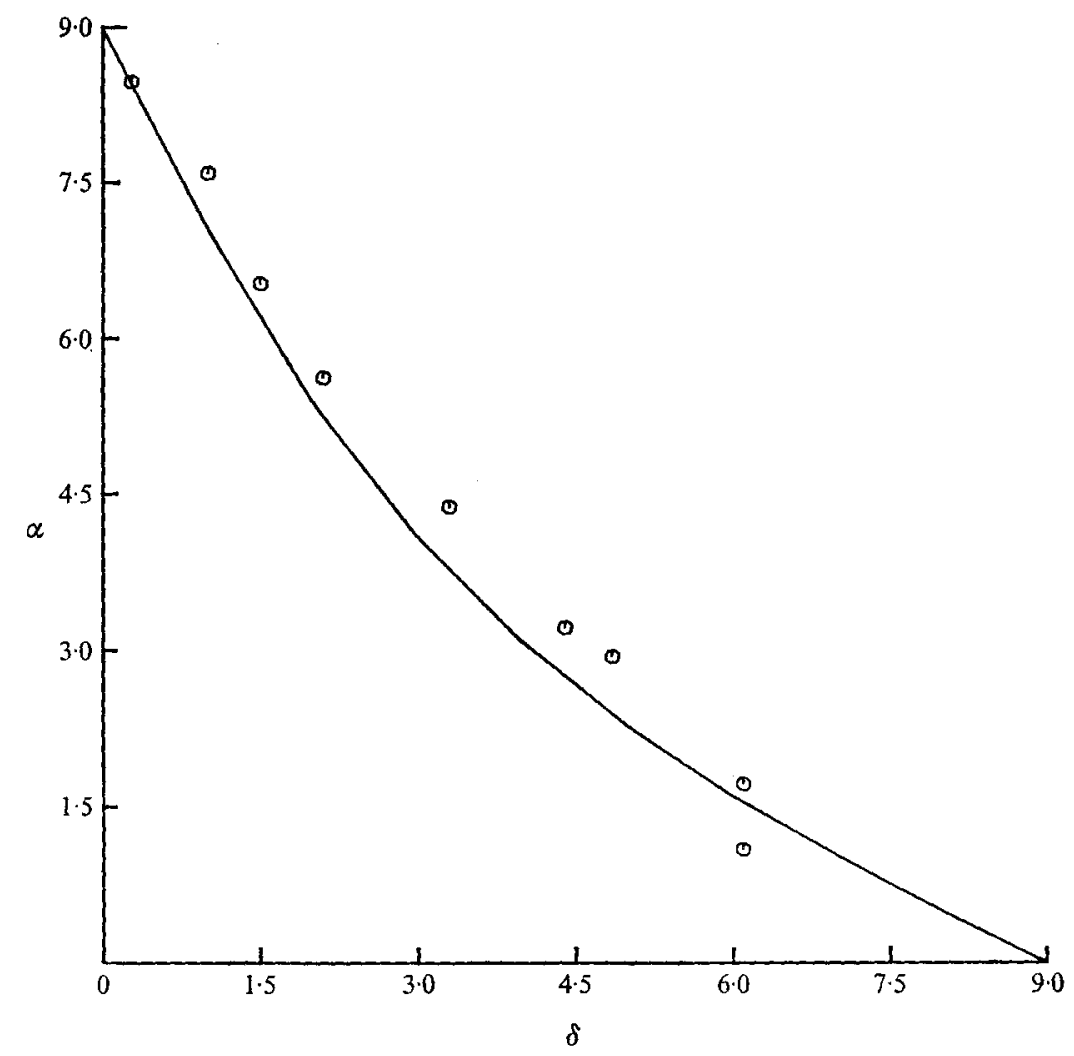

Figure 9. Measured values of the inclination $\delta$ of the particle axis to the horizontal $v s$. the inclination $\alpha$ of the path of motion to the particle axis. - - , calculated using (73); $\mathcal{O}$, experimental points, present investigation.

noted that figure 10 was, in fact, taken quite near the top of the tank since the re-orientation towards the vertical equilibrium position was generally complete by the midplane of the column. The re-orientation to vertical provides a strong confirmation of one of the principal predictions of the present theory. A more quantitative comparison of experimental results and the theoretical predictions of (47) will be presented in a forthcoming communication.

A second set of experiments was carried out which was intended to test the theoretical prediction that the terminal velocity of a vertically oriented cone would be smaller with its rounded end in the direction of motion than with its pointed end leading. The chief difficulty experienced with these experiments resulted from the fact that the point-down orientation was only marginally stable for the cones which we used, so that the particle showed a strong tendency to flip over to the configuration with the rounded end leading. Only those few particles which were essentially perfectly vertical at the beginning of the experiments actually remained in the point-down orientation to the midpoint of the tank, where the velocity measurements were made. The results were less conclusive than those of the previous paragraph. In the Newtonian $99.5 \%$ glycerine solution, no statistically significant differences in terminal velocity could be 


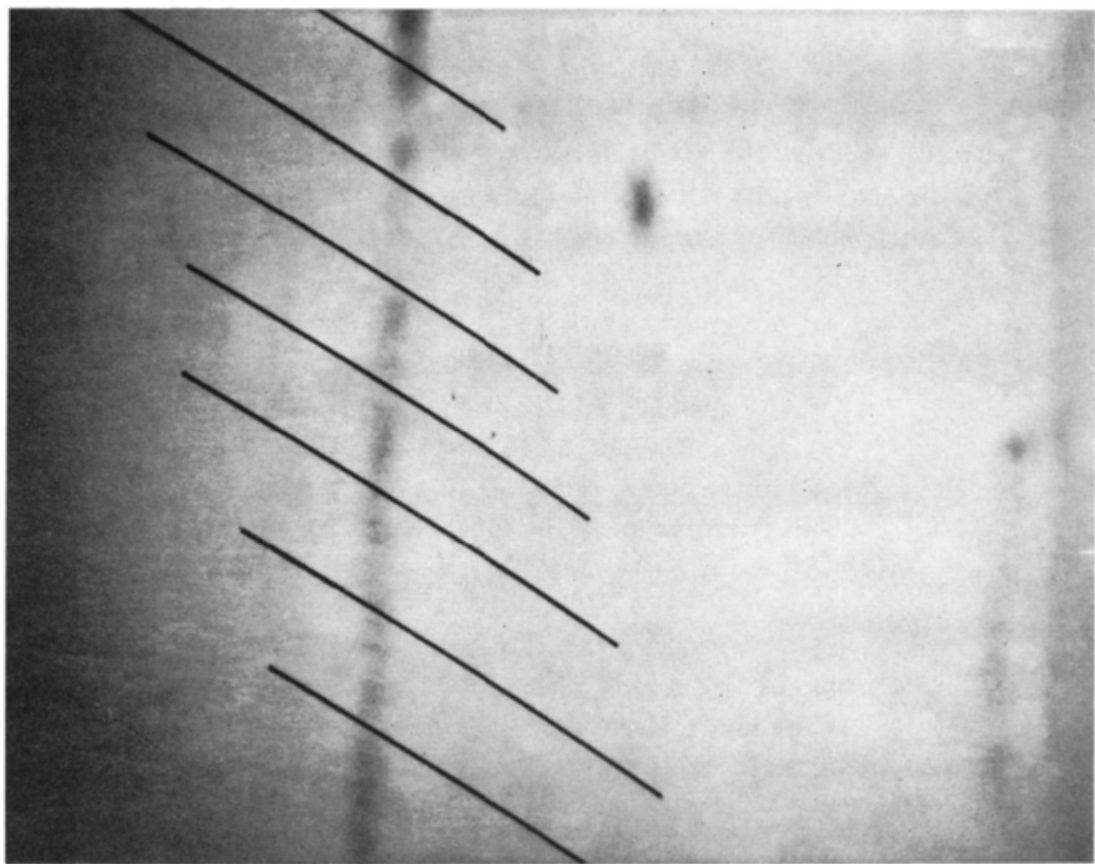

Fravae 8. Multiple-imago photograph of a slender rod-like particlo $(r=66)$ sedimenting through $99 \cdot 5 \%$ glycerine.

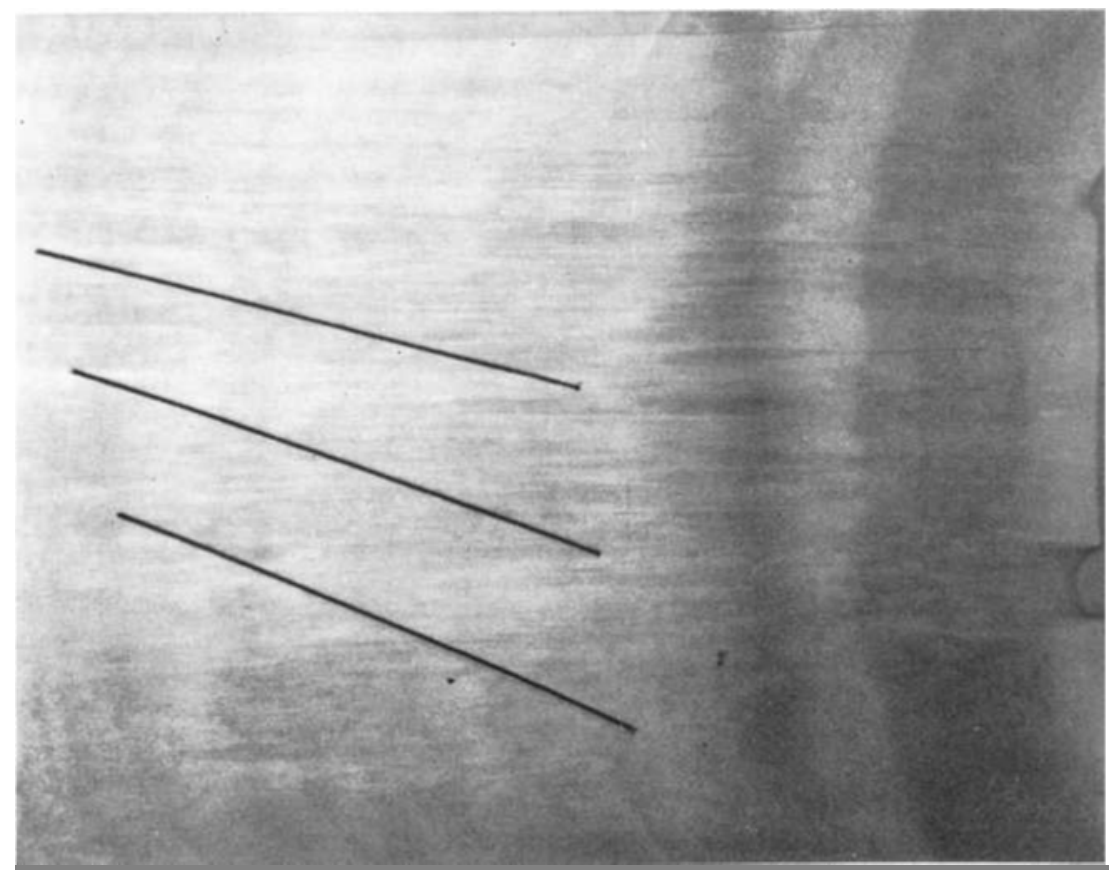

Fiture 10. Multiple-image photograph of a slender rod-like particle $(r=66)$ sedimenting through $0.5 \%$ Separan AP30 in water. 

ascertained between the point-up and point-down configurations. In Separan, on the other hand, a slight difference was apparent, with the particle falling more slowly in the point-up configuration as predicted by the theory. However, it must be noted that the difference was small, only marginally greater than the maximum variations observed from run to run with a fixed orientation. We hope to improve the accuracy of these results in a future investigation.

\section{REFERENCES}

Bartram, E. \& Mason, S. G. 1974 To be published.

BATChetor, G. K. 1970 J. Fluid Mech. 44, 419.

Brtndiey, G. \& Broadbent, J. M. 1973 Rheol. Acta, 12, 48.

Bruce, C. \& Schwarz, W. H. 1969 J. Polymer Sci. 7, 909.

Casweld, B. \& Schwarz, W. H. 1962 J. Fluid Mech. 13, 417.

Cox, R. G. 1970 a J. Fluid Mech. 44, 791.

Cox, R. G. 19706 J. Fluid Mech. 45, 625.

Cox, R. G. \& BrenNer, H. 1968 Chem. Engng Sci. 23, 147.

Frankel, N. A. \& ACrivos, A. 1970 J. Fluid Mech. 44, 65.

Gauthier, F., Goldsmith, H. L. \& Mason, S. G. 1971 Rheol. Acta, 10, 344.

GIESEkUS, H. 1963 Rheol. Acta, 3, 59.

Hinch, E. J. \& LeaL, L. G. 1972 J. Fluid Mech. 52, 683.

Ho, B. P. \& Leat, L. G. $1974 J$. Fluid Mech. 65, 365.

JEFFery, G. B. 1922 Proc. Roy. Soc. A 102, 161.

Karnis, A.\& Mason, S. G. 1967 Trans. Soc. Rheol. 10, 571.

Leat, L. G. \& Hinch, E. J. 1971 J. Fluid Mech. 46, 685.

LEAL, L. G. \& HiNCH, E. J. 1972 J. Fluid Mech. 55, 745.

LEsure, F. M. 1961 Quart. J. Mech. Appl. Math. 14, 36. .

Lin, C., Peery, J. H. \& Schowatter, W. R. 1970 J. Fluid Mech. 44, 1.

Mestre, N. J. DE 1973 J. Fluid Mech. 58, 641.

Olabisi, O. \& Williams, M. C. 1972 Trans. Soc. Rheol. 16, 727.

SaffMan, P. G. 1956 J. Fluid Mech. 1, 540.

Skoog, J., Leat, L. G. \& Acrivos, A. 1971 J. Chem. Engng, 49, 569.

TANner, R. I. 1970 Trans. Soc. Rheol. 14, 483.

Tillett, J. P. K. 1970 J. Fluid Mech. 44, 401.

WaLes, J. L. S. \& Philippoff, W. 1973 Rheol. Acta, 12, 25.

WeIsSENBERG, K. 1947 Nature, 159, 310. 